\title{
Análisis del período y desplazamiento de edificios de hormigón armado considerando distintos grados de rigidez en sus elementos resistentes
}

\author{
Period and displacement analysis of reinforced concrete buildings with different degrees of \\ stiffness in their resistance elements \\ Fecha de entrega: 14 de octubre 2015 \\ Fecha de aceptación: 23 de marzo 2016
}

\section{Constanza López y Juan Music}

Departamento de Ingeniería Civil, Universidad Católica del Norte, Av. Angamos 0610, Antofagasta, Chile, constanza.vlopez@gmail.com,jmusic@ucn.cl

En este trabajo se estudia principalmente la relación entre los periodos de mayor masa traslacional en condiciones denominadas agrietadas y no agrietadas, para edificios altos de hormigón armado (mayor a 10 pisos) ubicados en la ciudad de Antofagasta, Chile. La metodología consiste en determinar los períodos de los modos con mayor masa traslacional y los desplazamientos a nivel de techo mediante un análisis sísmico según NCh433 Of.96 Mod.2009/DS.61 considerando las secciones brutas de los elementos (condición llamada no agrietada). Posteriormente, se modifica la rigidez a flexión de los muros estructurales mediante factores de reducción del momento de inercia de las secciones brutas para considerar el agrietamiento (condición llamada agrietada). Estos factores de reducción corresponden a diferentes criterios recomendados por la comunidad científica. Los resultados obtenidos son comparados entre los distintos modelos y también con lo señalado por el Decreto 61, en relación al valor del periodo agrietado y el desplazamiento de diseño a nivel de techo. Se concluye que, a excepción de uno de los criterios utilizados (modelo E de Doepker), lo señalado por el Decreto 61 está por el lado de la seguridad, ya que la relación entre los periodos agrietados y no agrietados de los edificios estudiados, en general, es inferior a 1.5. De igual manera, los desplazamientos de techo resultan ser menores a los determinados con la expresión dada por el Decreto 61.

Palabras clave: periodo agrietado, desplazamiento de techo, NCh433 Of.96 Mod.2009/Decreto 61
This paper studies mainly the relationship between the fundamental translational periods of vibration, in tall reinforced concrete building (more than ten floors) located in Antofagasta, Chile, for both conditions, cracked and uncracked. For that purpose, the fundamental translational periods of vibration and also the roof displacement were calculated using the modal spectral analysis proposed in the Chilean seismic code NCh433 Of.96 Mod.2009/D.S.61, considering the gross section (uncracked condition). Afterward, the flexural stiffness of structural walls was modified applying reduction factors to the moment of inertia to consider the cracked condition. These reduction factors were taken from different recommendations given by the scientific community to take into account cracking in structural walls. Results for the different models were compared among them and with what is specified in the DS 61 with respect to periods and roof displacement. Finally, it is concluded that, in general, the ratio between periods for the cracked and uncracked are less than 1.5; except for the model E (Doepker). Similarly, the roof displacements are smaller than that specified by the formula included in the DS 61

Keywords: cracked period, roof displacement, NCh433 Of.96 Mod.2009/Decree 61

\section{Introducción}

El sismo del 27 de febrero de 2010 en Chile de magnitud momento 8.8 produjo, en general, un satisfactorio comportamiento sísmico de las estructuras construidas en los últimos 25 años. Sin embargo, unos pocos edificios de hormigón armado (menos del 1\%) presentaron importantes daños estructurales (Lagos et al., 2012). Los catastros realizados después del sismo en las ciudades de Santiago, Viña del Mar y Concepción mostraron daños en muros esbeltos con secciones $\mathrm{T}$ y rectangulares sometidos a esfuerzos de compresión y cargas laterales. Los elementos dañados se localizaron fundamentalmente en el primer 
piso y en el primer subterráneo, donde habitualmente se ubican los estacionamientos para vehículos. En ellos fue posible observar una grieta a lo largo del alma de los muros, descascaramiento del hormigón, pandeo de barras longitudinales y una fractura abrupta de barras en algunos casos. Estas fallas se originaron debido a grandes solicitaciones en las zonas más comprimidas de los muros sumadas a problemas en el detallamiento de elementos de borde y en la disposición del refuerzo transversal. Esto último no permitió proveer un confinamiento adecuado al hormigón ni restricción al pandeo de las barras longitudinales (Alfaro, 2013; Wallace et al., 2012).

Producto del terremoto antes señalado el Ministerio de Vivienda y Urbanismo (MINVU) conformó un panel de expertos en materia relativas al diseño sísmico de edificios. Este comité estudió las modificaciones necesarias tanto para el código sísmico como para la normativa de diseño de hormigón armado, recogiendo la experiencia de los daños observados en edificios en la zona centro y sur del país. Como resultado de este trabajo en febrero de 2011 se dictaron dos decretos. Por un lado se promulgó el Decreto 117 (MINVU, 2011a), que fija el diseño sísmico de edificios, modificando fundamentalmente la clasificación sísmica de suelos de fundación y el espectro de diseño que hasta la fecha era regulado por la norma $\mathrm{NCh} 433$ of 96 Mod. 2009 (INN, 2010). Al mismo tiempo se promulgó el Decreto 118 (MINVU, 2011b), que fija los requisitos de diseño y cálculo para estructuras de hormigón armado. Estos decretos fueron reemplazados, en noviembre de 2011 por los Decretos 60 y 61, actualmente vigentes (MINVU, 2011c,d).

El Decreto 61, entre otros aspectos, establece que "para efecto de diseño de estructuras de hormigón armado, el desplazamiento lateral de diseño en el techo, $\delta_{u}$, se debe considerar igual a la ordenada del espectro elástico de desplazamiento $S_{\text {de }}$, para un $5 \%$ de amortiguamiento respecto del crítico, correspondiente al período de mayor masa traslacional en la dirección del análisis, multiplicada por un factor igual a $1.3\left(\delta_{\mathrm{u}}=1.3 S_{\mathrm{de}}\left(T_{\mathrm{ag}}\right)\right)$ ". Para la determinación del período de mayor masa traslacional en la dirección de análisis, señala dos formas: $i$ ) determinar $T_{\mathrm{ag}}$ considerando en su cálculo la influencia del acero y la pérdida de rigidez debida al agrietamiento del hormigón en la rigidez elástica inicial y ii) si el período ha sido calculado con las secciones brutas, es decir, sin considerar la influencia del acero y la pérdida de la rigidez debido al agrietamiento del hormigón, el período de mayor masa traslacional en la dirección de análisis de la estructura se puede aproximar a 1.5 veces al calculado sin considerar estos efectos $\left(T_{\mathrm{ag}}=1.5 T_{\mathrm{n}}\right)$.

En este trabajo se seleccionan ocho edificios de hormigón armado ubicados en la ciudad de Antofagasta y se determina $T_{\text {ag }}$ por ambas formas señaladas en el Decreto $61 \mathrm{y}$ descrita anteriormente. Adicionalmente se calculan los valores del desplazamiento lateral de diseño en el techo, $\delta_{u}$. Para ello se realiza el análisis elástico dinámico, según normativa vigente nacional, para ambas direcciones de análisis. Para evaluar $T_{\text {ag }}$ según la primera forma indicada, se consideraron distintos criterios de agrietamiento en los muros estructurales. En este trabajo se analizan seis modelos, los cuales modifican la rigidez a flexión no fisurada de los muros por un factor reductor según expresiones empíricas propuestas por códigos e investigadores.

Algunas recomendaciones proveen un único y uniforme factor reductor de la rigidez determinada con las secciones brutas, tales como el código ACI 318 (2008) y FEMA 356 (2000). Otras expresiones más desarrolladas entregan recomendaciones relacionadas con la carga axial (Paulay y Priestley, 1992; Adebar e Ibrahim, 2002) y otras son expresiones en función de la demanda de desplazamientos (Doepker, 2008).

Con este estudio se logra para los edificios analizados: $i$ ) comparar los períodos agrietados y no agrietados (según los distintos modelos elegidos) y considerando distintos niveles de muros agrietados; ii) determinar expresión que relacione el período no agrietado y agrietado para cada modelo elegido y iii) comparar la relación entre los desplazamientos de techo determinado con la estructura agrietada y lo indicado en el Decreto 61.

\section{Rigidez efectiva de elementos de hormigón armado}

Se exponen a continuación diferentes criterios existentes para considerar la rigidez efectiva de elementos estructurales, mediante factores de reducción aplicados a las propiedades de las secciones brutas. Estos son: 


\section{Requisitos para hormigón estructural - ACI 318}

El código ACI 318 desde la edición del año 1999 hasta la del 2014, ha considerado la disminución de rigidez debido a las deformaciones que se esperan producir con los niveles cercanos a la carga última. Para ello, plantea tres alternativas que determinan el momento de inercia efectivo, las cuales se pueden utilizar para análisis elásticos (ACI 318, 2014).

Alternativa 1: por las propiedades de las secciones definidas de acuerdo a la Tabla 1.

Tabla 1: Momento de inercia efectivo propuesto por el código ACI 318 (2014)

\begin{tabular}{|c|c|c|c|}
\hline \multicolumn{2}{|c|}{$\begin{array}{l}\text { Miembros y } \\
\text { condición }\end{array}$} & $\begin{array}{l}\text { Momento de } \\
\text { inercia }\end{array}$ & $\begin{array}{l}\text { Área de la sección } \\
\text { transversal }\end{array}$ \\
\hline \multicolumn{2}{|c|}{ Columnas } & $0.7 I_{\mathrm{g}}$ & \multirow{5}{*}{$A_{\mathrm{g}}$} \\
\hline \multirow{2}{*}{ Muros } & $\begin{array}{l}\text { No } \\
\text { fisurados }\end{array}$ & $0.7 I_{\mathrm{g}}$ & \\
\hline & Fisurados & $0.35 I_{\mathrm{g}}$ & \\
\hline \multicolumn{2}{|l|}{ Vigas } & $0.35 I_{\mathrm{g}}$ & \\
\hline \multicolumn{2}{|c|}{$\begin{array}{l}\text { Placas planas y } \\
\text { losas planas }\end{array}$} & $0.25 I_{\mathrm{g}}$ & \\
\hline
\end{tabular}

Alternativa 2: el 50\% de los valores de rigidez basados en las propiedades de la sección bruta $\left(I_{\mathrm{e}}=0.5 I_{\mathrm{g}}\right)$.

Alternativa 3: otras expresiones sugeridas para determinar el momento de inercia efectivo (incorporadas en la versión del año 2008) son mostradas en la Tabla 2.

$I_{\mathrm{g}}$ : momento de inercia de la sección bruta del elemento respecto al eje que pasa por el centroide sin tener en cuenta el acero de refuerzo, en $\mathrm{mm}^{4}, I_{\mathrm{e}}$ : momento de inercia efectivo en $\mathrm{mm}^{4}, A_{\mathrm{st}}$ : área total del refuerzo longitudinal en $\mathrm{mm}^{2}, A_{\mathrm{g}}$ : área bruta de la sección de hormigón en $\mathrm{mm}^{2}$ : $P_{\mathrm{u}}, M_{\mathrm{u}}$ : fuerza axial y momento mayorado en $\mathrm{N}$ y $\mathrm{Nmm}$, $P_{0}$ : resistencia axial nominal para una excentricidad igual a cero, en $\mathrm{N}, \rho$ : cuantía de refuerzo $A_{\mathrm{s}}$ evaluada sobre el área $b d, b_{\mathrm{w}}$ : ancho del alma o diámetro de la sección circular en $\mathrm{mm}, d$ : distancia desde la fibra extrema en compresión hasta el centroide del refuerzo longitudinal en tracción en $\mathrm{mm}, h$ : espesor total o altura de un elemento en $\mathrm{mm}$.

\section{FEMA 356}

FEMA 356 (2000) recomienda que la rigidez de los elementos de una estructura puede ser disminuida por los valores mostrados en la Tabla 3.

Tabla 3: Rigidez efectiva propuesta por el FEMA 356 (2000)

\begin{tabular}{|l|c|c|c|}
\hline Elementos & $\begin{array}{c}\text { Rigidez } \\
\text { a flexión }\end{array}$ & $\begin{array}{c}\text { Rigidez } \\
\text { al corte }\end{array}$ & $\begin{array}{c}\text { Rigidez } \\
\text { axial }\end{array}$ \\
\hline Vigas-no pretensadas & $0.5 E_{\mathrm{c} g} I_{\mathrm{g}}$ & $0.4 E_{\mathrm{c}} A_{\mathrm{w}}$ & - \\
\hline Vigas-pretensadas & $E_{\mathrm{c} g} I_{\mathrm{g}}$ & $0.4 E_{\mathrm{c}} A_{\mathrm{w}}$ & - \\
\hline $\begin{array}{l}\text { Columnas a compresión } \\
\text { con cargas de diseño } \\
\leq 0.5 A_{\mathrm{g}} f_{\mathrm{c}}\end{array}$ & $0.7 E_{\mathrm{c} g} I_{\mathrm{g}}$ & $0.4 E_{\mathrm{c}} A_{\mathrm{w}}$ & $E_{\mathrm{c}} A_{\mathrm{g}}$ \\
\hline $\begin{array}{l}\text { Columnas a compresión } \\
\text { con cargas diseño } \\
\leq 0.3 A_{\mathrm{g}} f_{\mathrm{c}} \text { o con tracción }\end{array}$ & $0.5 E_{\mathrm{c} g}$ & $0.4 E_{\mathrm{c}} A_{\mathrm{w}}$ & $E_{\mathrm{s}} A_{\mathrm{s}}$ \\
\hline $\begin{array}{l}\text { Muros - no agrietados } \\
\text { (con inspección) }\end{array}$ & $0.8 E_{\mathrm{c} g} I_{\mathrm{g}}$ & $0.4 E_{\mathrm{c}} A_{\mathrm{w}}$ & $E_{\mathrm{c}} A_{\mathrm{g}}$ \\
\hline Muros agrietados & $0.5 E_{\mathrm{c} g}$ & $0.4 E_{\mathrm{c}} A_{\mathrm{w}}$ & $E_{\mathrm{c}} A_{\mathrm{g}}$ \\
\hline Losas no pretensadas & - & $0.4 E_{\mathrm{c}} A_{\mathrm{g}}$ & - \\
\hline Losas pretensadas & - & $0.4 E_{\mathrm{c}} A_{\mathrm{g}}$ & - \\
\hline
\end{tabular}

$A_{\mathrm{g}}$ : área bruta horizontal, $A_{\mathrm{s}}$ : área del refuerzo, $A_{\mathrm{w}}$ : área del alma de la sección bruta, $E_{\mathrm{c}}$ : módulo de elasticidad del hormigón

\section{Adebar e Ibrahim}

Adebar e Ibrahim (2002) propusieron las siguientes expresiones para la rigidez efectiva a flexión de muros de hormigón armado para utilizar en análisis sísmicos lineales.

Tabla 2: Expresiones alternativas para momento de inercia según el código ACI 318 (2014)

\begin{tabular}{|l|c|c|c|}
\hline \multirow{2}{*}{ Miembro } & \multicolumn{3}{|c|}{ Valor alternativo de $I$ para análisis elástico } \\
\cline { 2 - 4 } Columnas y muros & Mínimo & $I$ & Máximo \\
\hline $\begin{array}{l}\text { Vigas, placas planas y } \\
\text { losas planas }\end{array}$ & $0.35 I_{\mathrm{g}}$ & $I_{e}=\left(0.8+25 \frac{A_{s t}}{A_{g}}\right)\left(1-\frac{M_{u}}{P_{u} h}-0.5 \frac{P_{u}}{P_{o}}\right) I_{g}$ & $0.875 I_{\mathrm{g}}$ \\
\hline
\end{tabular}


Momento efectivo de inercia en muros no agrietados:

$$
I_{e}=\left(0.6+\frac{P_{u}}{f_{c}^{\prime} A_{g}}\right) I_{g} \leq I_{g}
$$

Momento efectivo de inercia en muros agrietados:

$$
I_{e}=\left(0.2+2.5 \frac{P_{u}}{f_{c}^{\prime} A_{g}}\right) I_{g} \leq 0.7 I_{g}
$$

\section{Paulay y Priestley}

Paulay y Priestley (1992) definen que la rigidez efectiva de un muro en voladizo sometido predominantemente a deformaciones por flexión, puede ser determinada mediante el momento de inercia efectivo $I_{\mathrm{e}}$ de la sección que alcanza la primera fluencia en la fibra extrema, mediante la siguiente expresión:

$$
I_{e}=\left(\frac{100}{f_{y}}+\frac{P_{u}}{f_{c}^{\prime} A_{g}}\right) I_{g}
$$

donde $P_{\mathrm{u}}$ es la carga axial que actúa en el muro durante un sismo (es tomada positiva para compresión y negativa en tracción); en $\mathrm{N}, f_{\mathrm{y}}$ es la tensión de fluencia del refuerzo en $\mathrm{MPa}, f_{\mathrm{c}}$ ' es la resistencia a compresión del hormigón en MPa y $A_{\mathrm{g}}$ es el área horizontal bruta del elemento en $\mathrm{mm}^{2}$.

\section{Doepker}

Doepker (2008) estudió los distintos métodos existentes para estimar la rigidez y el amortiguamiento efectivo en estructuras compuestas de muros de hormigón armado. En base a ello propone un nuevo método para predecir la rigidez efectiva en función del desplazamiento de la estructura.

$$
I_{e}=\left[0.8 e^{0.05 \frac{\Delta_{\text {roof }}}{H}}-0.7\left(\frac{\Delta_{\text {roof }}}{H}\right)^{0.2}\right] I_{g}
$$

donde $\Delta_{\text {roof }}$ es el desplazamiento del techo y $H$ es la altura del edificio. Además, el autor concluye que usar una relación de amortiguamiento del $3 \%$ produce los menores errores.

Las Figuras 1a) y 1b) resumen las distintas expresiones que se presentaron para determinar la rigidez efectiva de muros estructurales, tanto en condiciones agrietadas como no agrietadas.
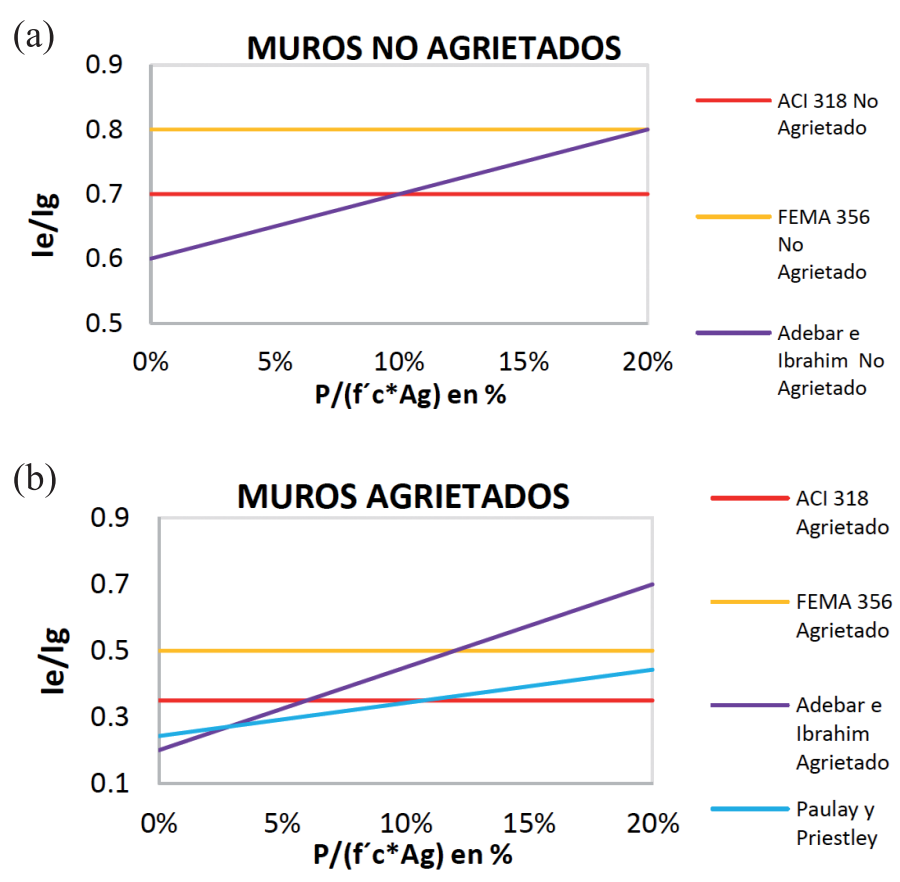

Figura 1: Gráficos de las expresiones para determinar la rigidez efectiva de muros: a) rigidez efectiva en muros no agrietados y b) rigidez efectiva en muros agrietados

\section{Edificios y modelos de agrietamiento para el estudio}

Las estructuras analizadas en este estudio corresponden a edificios destinados a uso habitacional ubicados en la ciudad de Antofagasta (Tabla 4). Todos ellos están estructurados en base a muros de hormigón armado. Siete de los edificios tienen entre 13 y 18 pisos y están fundados en roca, mientras que el edificio más alto, que es el Pérez Zujovic, tiene 28 pisos y está fundado sobre suelo arenoso, para más detalles de los edificios ver Ríos (2006) y Cornejo (2012).

Tabla 4: Características generales de los edificios estudiados

\begin{tabular}{|l|c|c|c|c|c|}
\hline Edificio & Año & $\begin{array}{c}\text { Altura, } \\
\mathrm{m}\end{array}$ & $\begin{array}{c}\text { Área } \\
\text { total, } \mathrm{m}^{2}\end{array}$ & Pisos & $\begin{array}{c}\text { Subterrá- } \\
\text { neos }\end{array}$ \\
\hline Barlovento & 2009 & 41.42 & 5342 & 16 & 1 \\
\hline Grecia & 1999 & 33.7 & 2305 & 13 & 1 \\
\hline Las Palmas & 1997 & 38.58 & 6580 & 15 & 1 \\
\hline Los Sauces & 1999 & 37.85 & 15215 & 15 & 0 \\
\hline Mar del Sur & 1993 & 33.2 & 1614 & 13 & 0 \\
\hline $\begin{array}{l}\text { Mirador del } \\
\text { Sur }\end{array}$ & 1999 & 41.41 & 6054 & 16 & 1 \\
\hline Pérez Zujovic & 1978 & 74.49 & 14104 & 28 & 1 \\
\hline Plaza Foresta & 2002 & 46.88 & 5501 & 18 & 1 \\
\hline
\end{tabular}


López, C. y Music, J. (2016). Análisis del período y desplazamiento de edificios de hormigón armado considerando distintos grados de rigidez en sus elementos resistentes. Obrasy Proyectos 19, 33-47

La Figura 2 muestra fotografías de los ocho edificios analizados junto con sus períodos de mayor masa traslacional en condiciones no agrietadas y el desplazamiento de diseño de techo en cada dirección de análisis determinado según lo dispuesto en el D.S. 61. El significado de cada término es el siguiente: $T_{n x}^{*}, T_{n y}^{*}$ ,corresponden al período del modo con mayor masa traslacional en condiciones no agrietadas para sismo en la dirección $X$ e $Y, \delta_{\mathrm{ux}}, \delta_{\mathrm{uy}}$ corresponden al desplazamiento de diseño de techo para sismo en la dirección $X$ e $Y$. La elección de las direcciones $X$ e $Y$ depende de cada edificio y son mutuamente perpendiculares. Sin embargo, es importante mencionar, que no siempre la dirección $X$ elegida coincide con la dirección de mayor rigidez del edificio.

Barlovento

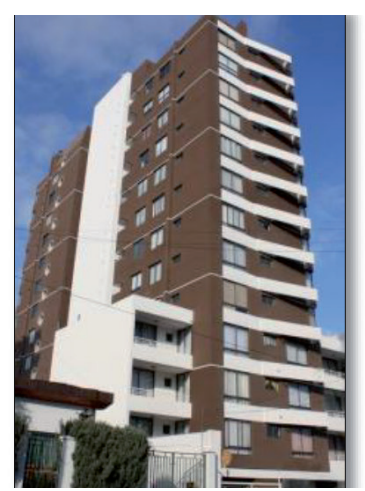

$T_{\mathrm{nx}}^{*}=0.368 \mathrm{~s}, T_{\mathrm{ny}}^{*}=0.74 \mathrm{~s}$

$\delta_{\mathrm{ux}}=5.3 \mathrm{~cm}, \delta_{\mathrm{uy}}=12.1 \mathrm{~cm}$

Mar del Sur

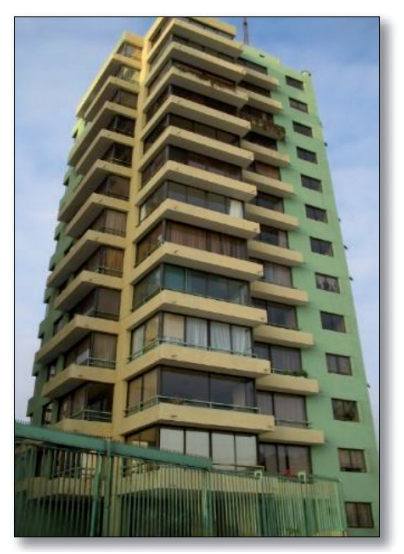

$T_{\mathrm{nx}}^{*}=0.463 \mathrm{~s}, T_{\mathrm{ny}}^{*}=0.712 \mathrm{~s}$

$\delta_{\mathrm{ux}}=11.6 \mathrm{~cm}, \delta_{\mathrm{uy}}=6.9 \mathrm{~cm}$
Grecia

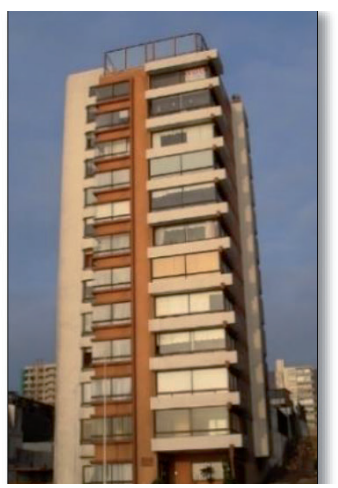

$T_{\mathrm{nx}}^{*}=0.496 \mathrm{~s}, T_{\mathrm{ny}}^{*}=0.397 \mathrm{~s}$

$\delta_{\mathrm{ux}}=7.5 \mathrm{~cm}, \delta_{\mathrm{uy}}=5.8 \mathrm{~cm}$

Mirador del Sur

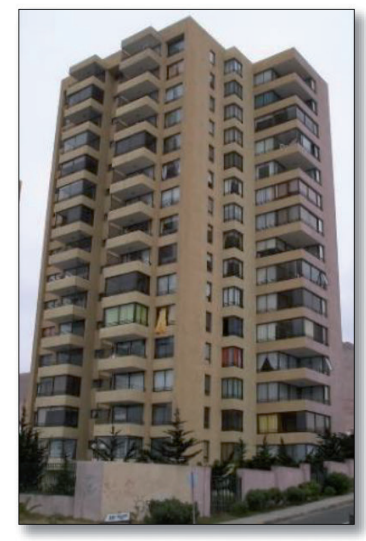

$T_{\mathrm{nx}}^{*}=0.533 \mathrm{~s}, T_{\mathrm{ny}}^{*}=0.76 \mathrm{~s}$

$\delta_{\text {ux }}=8.2 \mathrm{~cm}, \delta_{\text {uy }}=12.5 \mathrm{~cm}$

\section{Modelos de agrietamiento utilizados en el estudio}

En esta sección se explican los distintos criterios de agrietamiento seleccionados en este estudio, los que dan origen a los diferentes modelos utilizados y aplicados a los edificios seleccionados.

- Modelo A: utiliza la expresión (3) de Paulay y Priestley, la cual es aplicada solo a los muros considerados agrietados. Para los muros no agrietados se ha considerado utilizar un factor único de reducción igual a 0.8 .

- Modelo B: utiliza la expresión (2) de Adebar e Ibrahim para los muros agrietados. Mientras que para los muros no agrietados se ha utilizado un factor reductor

Las Palmas

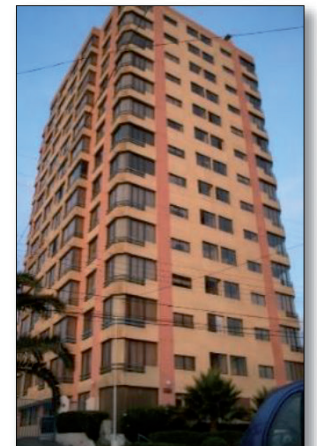

$T_{\mathrm{nx}}^{*}=0.478 \mathrm{~s}, T_{\mathrm{ny}}^{*}=0.53 \mathrm{~s}$

$\delta_{\mathrm{ux}}=7.2 \mathrm{~cm}, \delta_{\mathrm{uy}}=8.1 \mathrm{~cm}$

\section{Perez Zujovic}

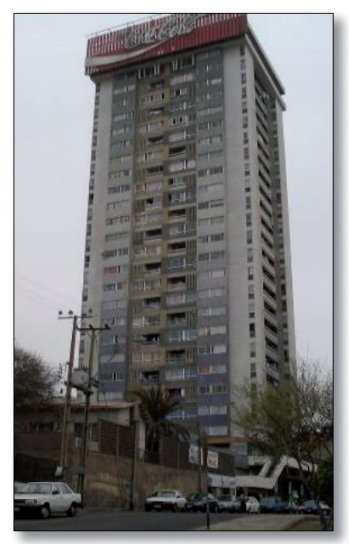

$T_{\mathrm{nx}}^{*}=1.143 \mathrm{~s}, T_{\mathrm{ny}}^{*}=1.624 \mathrm{~s}$

$\delta_{\mathrm{ux}}=27.6 \mathrm{~cm}, \delta_{\mathrm{uy}}=34.2 \mathrm{~cm}$
Los Sauces

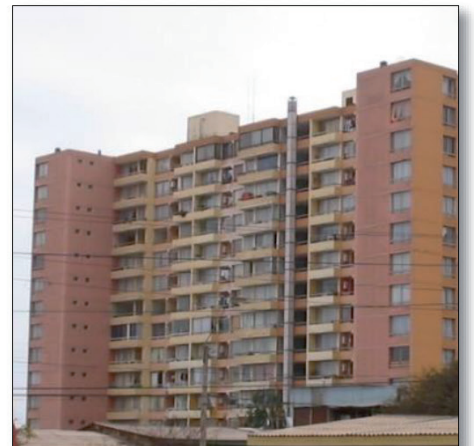

$T_{\mathrm{nx}}^{*}=0.35 \mathrm{~s}, T_{\mathrm{ny}}^{*}=0.451 \mathrm{~s}$

$\delta_{\mathrm{ux}}=5 \mathrm{~cm}, \delta_{\mathrm{uy}}=6.7 \mathrm{~cm}$

Plaza Foresta

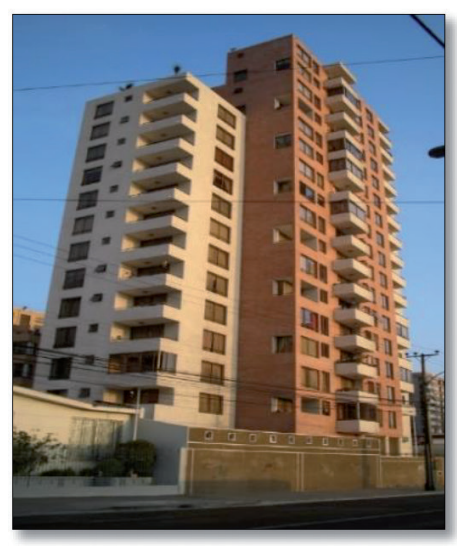

$T_{\mathrm{nx}}^{*}=0.469 \mathrm{~s}, T_{\mathrm{ny}}^{*}=0.743 \mathrm{~s}$

$\delta_{\mathrm{ux}}=7 \mathrm{~cm}, \delta_{\mathrm{uy}}=12.2 \mathrm{~cm}$

Figura 2: Fotos de edificios estudiados, períodos y desplazamientos de diseño 
de 0.7 (valor medio del recomendado para muros no agrietados).

- Modelo C: utiliza la recomendación del código ACI 318 que considera que los muros agrietados son modificados por un factor de 0.35 , mientras que el resto de los muros es modificado por 0.7 .

- Modelo D: utiliza la recomendación del FEMA 356, donde todos los muros estructurales considerados como agrietados son modificados por el factor reductor 0.5 , mientras que el resto de los muros es modificado por 0.8 .

- Modelo E: La expresión de Doepker es un proceso iterativo, el cual consiste primero en modificar la rigidez de todos los muros (iniciando con $I_{\mathrm{e}}=0.8 I_{\mathrm{g}}$ ), luego determinar el desplazamiento a nivel de techo y continuar realizando este proceso hasta igualar la expresión (4) propuesta por el autor. La modificación de la rigidez se aplica a todos los muros del edificio por un único factor y el desplazamiento es obtenido en el centro de masa a nivel de techo (último nivel significativo). Además, se emplea una razón de amortiguamiento de 0.03 (Tabla 5).

Tabla 5: Resultados de reducción del momento de inercia modelo E

\begin{tabular}{|l|c|}
\hline Edificio & Factor de reducción utilizado \\
\hline Barlovento & 0.24 \\
\hline Grecia & 0.31 \\
\hline Las Palmas & 0.32 \\
\hline Los Sauces & 0.34 \\
\hline Mar del Sur & 0.28 \\
\hline Mirador del Sur & 0.32 \\
\hline Pérez Zujovic & 0.29 \\
\hline Plaza Foresta & 0.28 \\
\hline
\end{tabular}

- Modelo F: aplica la recomendación del código ACI 318 considerando un factor de reducción constante para todos los muros del edificio igual a 0.5 .

\section{Análisis y comparación de resultados}

Considerando que los daños ocurridos en los muros de hormigón armado, producto del terremoto del 27 de febrero de 2010 se localizaron en los muros esbeltos del primer subterráneo y primer piso (Wallace et al., 2012), es que se considera como muros agrietados aquellos muros esbeltos (relación de aspecto igual y mayor a 3 ) de los tres primeros niveles de pisos en todos los edificios estudiados, excepto el Pérez Zujovic, donde se considera cinco niveles de pisos de muros agrietados dado el mayor número de pisos respecto a los otros. Los muros restantes son considerados en condiciones no agrietados. A continuación se muestran los resultados obtenidos.

\section{Comparación del factor $\boldsymbol{k}$}

Con el objetivo de comparar la variación del período agrietado con lo dispuesto en el Decreto 61, se define el factor $k$ como la relación entre el período agrietado $T_{a g}^{*} \mathrm{y}$ no agrietado $T^{*}$.

$$
k=\frac{T_{a g(X, Y)}^{*}}{T_{(X, Y)}^{*}}
$$

En las Figuras 3 y 4 se muestran los valores obtenidos de $k$ para los edificios estudiados, los distintos modelos considerados y para ambas direcciones de análisis $(X, Y)$.

De los resultados obtenidos del factor $k$ se puede observar que en general la mayoría de los modelos entregan valores inferiores a 1.5. La excepción de estos resultados fueron obtenidos por el modelo $\mathrm{E}$, donde en varios edificios se obtuvo $k$ mayor a 1.5 , alcanzando valores de 1.84 . Por otro lado, los modelos A y B entregan resultados cercanos a 1.5, sobrepasando en algunos edificios dicho valor. En estos dos modelos, la rigidez efectiva utilizada en la mayoría de los muros agrietados adquiere el valor límite inferior propuesto por los autores, es decir, $0.25 I_{\mathrm{g}}$ en la expresión (3) y $0.2 I_{\mathrm{g}}$ en la expresión (2), representando de esta manera a los muros sin carga axial. Por otro lado, los análisis con el modelo $\mathrm{C}$ del código ACI mostraron resultados similares a los del modelo F para ambas direcciones de análisis.

\section{Correlación entre el período agrietado y no agrietado}

Se determina una correlación lineal entre el período agrietado y no agrietado para los seis modelos considerados. Los valores observados para realizar el ajuste incluyen los datos de ambas direcciones de análisis $X$ e $Y$. Una vez ajustada la recta de regresión de los modelos (mostradas en la Tabla 6), se tiene que el coeficiente de correlación $\mathrm{R}^{2}$ es superior a 0.96 . 


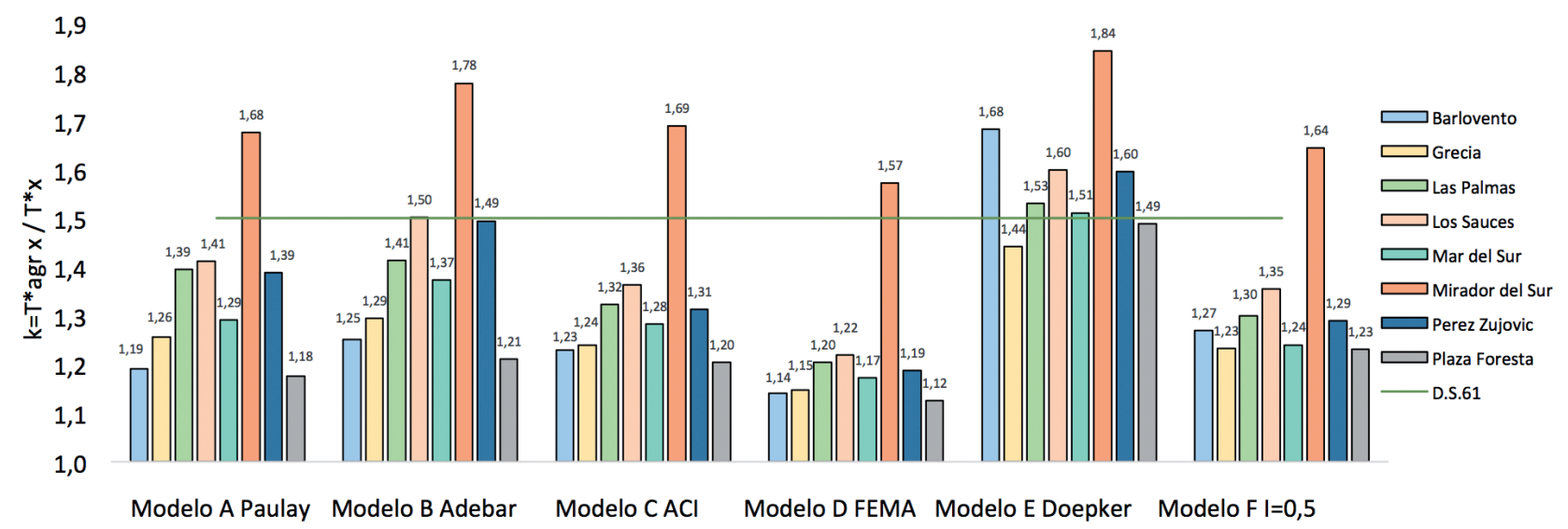

Figura 3: Relación $k$ para dirección de sismo $X$
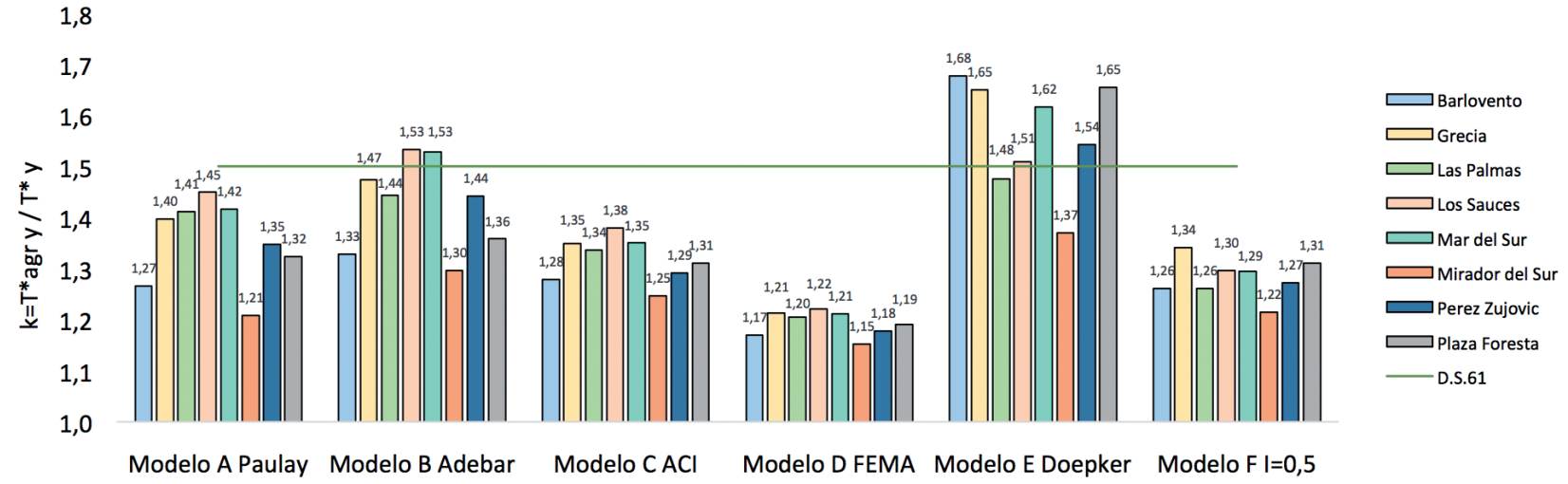

Figura 4: Relación $k$ para dirección de sismo $Y$

Tabla 6: Correlación lineal: período agrietado versus período no agrietado

\begin{tabular}{|c|c|c|}
\hline Modelos & Ajuste & $\mathrm{R}^{2}$ \\
\hline $\mathrm{A}$ & $T_{a g}^{*}=1.28 T^{*}+0.0347$ & 0.98 \\
\hline $\mathrm{B}$ & $T_{a g}^{*}=1.35 T^{*}+0.0448$ & 0.97 \\
\hline $\mathrm{C}$ & $T_{a g}^{*}=1.25 T^{*}+0.0302$ & 0.98 \\
\hline $\mathrm{D}$ & $T_{a g}^{*}=1.16 T^{*}+0.007$ & 0.99 \\
\hline $\mathrm{E}$ & $T_{a g}^{*}=1.55 T^{*}+0.011$ & 0.98 \\
\hline $\mathrm{F}$ & $T_{a g}^{*}=1.26 T^{*}+0.0164$ & 0.98 \\
\hline
\end{tabular}

\section{Comparación entre desplazamientos de techo}

El Decreto 61 establece que el desplazamiento lateral de diseño en el techo se debe considerar igual a la ordenada del espectro elástico de desplazamientos $S_{\mathrm{de}}$, para un 5\% de amortiguamiento respecto al crítico, correspondiente al período agrietado de mayor masa traslacional en la dirección de análisis, multiplicada por un factor igual a 1.3.

$\delta_{\mathrm{u}}=1.3 S_{\mathrm{de}}\left(T_{\mathrm{ag}}\right)$
Donde $T_{\text {ag }}$ es el período de mayor masa traslacional en la dirección de análisis, considerando en su cálculo la influencia del acero y la pérdida de rigidez debido al agrietamiento del hormigón en la rigidez elástica inicial. Si el período ha sido calculado con las secciones brutas, es decir, sin considerar la influencia del acero y la pérdida de la rigidez debido al agrietamiento del hormigón, el período de mayor masa traslacional en la dirección de análisis de la estructura se puede aproximar a 1.5 veces al calculado sin considerar estos efectos $\left(T_{\mathrm{ag}}=1.5 T_{\mathrm{n}}\right)$. El espectro elástico de desplazamientos $S_{\mathrm{de}}\left(T_{\mathrm{n}}\right)$ se calcula según la siguiente expresión:

$$
S_{d e}\left(T_{n}\right)=\frac{T_{n}^{2}}{4 \pi^{2}} \alpha A_{0} C_{d}^{*}
$$

donde los valores de $C_{d}^{*}$ son indicados en la Tabla 7 para los suelos tipo A y B. Además, en la Figura 5 se muestran los valores del desplazamiento de techo de diseño para ambos tipos de suelo y zona sísmica 3 . 
Tabla 7: Valores del coeficiente $C_{d}^{*}$

\begin{tabular}{|c|c|c|}
\hline $\begin{array}{c}\text { Tipo } \\
\text { de } \\
\text { suelo }\end{array}$ & $C_{d}^{*}$ & Rango de períodos \\
\hline & 1.0 & $T_{n} \leq 0.23 \mathrm{~s}$ \\
$\mathrm{~A}$ & $-0.055 T_{n}^{2}+0.36 T_{n}+0.92$ & $0.23<T_{n} \leq 0.252 \mathrm{~s}$ \\
& $0.08 T_{n}^{2}-0.9 T_{n}+0.324$ & $2.52<T_{n} \leq 5 \mathrm{~s}$ \\
\hline & 1.0 & $T_{n} \leq 0.47 \mathrm{~s}$ \\
$\mathrm{~B}$ & $0.95 T_{n}+0.55$ & $0.47<T_{n} \leq 2.02 \mathrm{~s}$ \\
& $0.065-0.75 T_{n}+3.72$ & $2.02<T_{n} \leq 5 \mathrm{~s}$ \\
\hline
\end{tabular}

Las Figuras 6 a 13 presentan los valores del desplazamiento de techo determinados en cada edificio en las direcciones de análisis $X$ e $Y$ una vez aplicado los modelos de

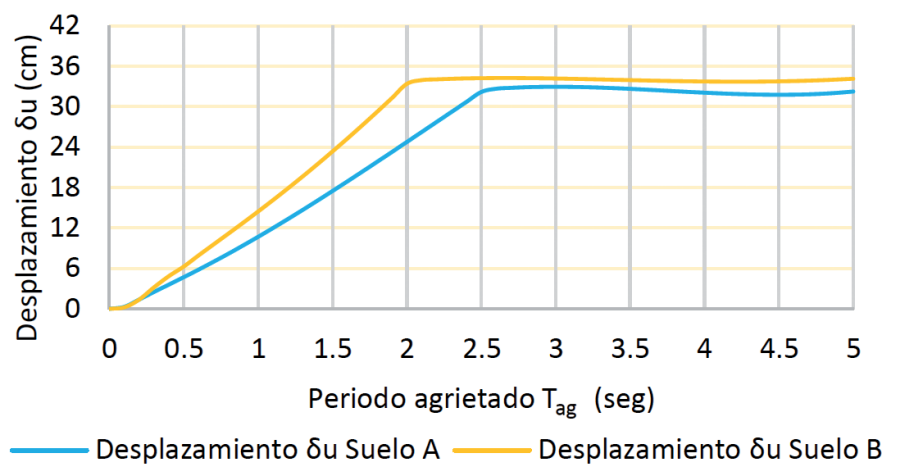

Figura 5: Desplazamiento de techo para suelos tipo A y B y zona sísmica 3

agrietamiento. En cada gráfico, además se incluye el desplazamiento de techo determinado por el D.S. 61.

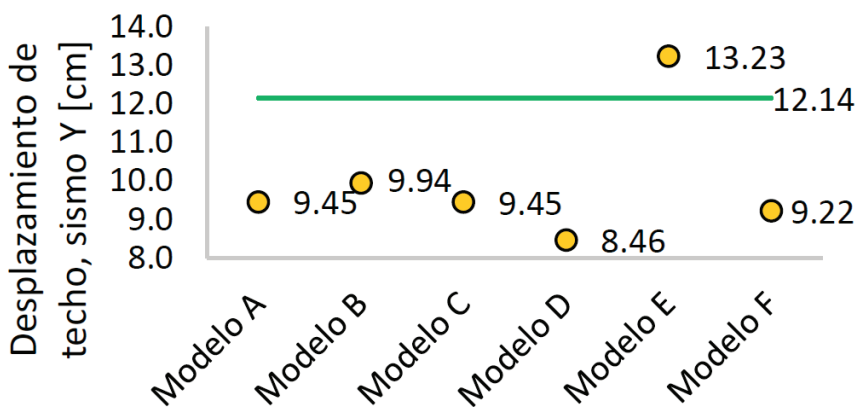

Figura 6: Desplazamiento de techo en el edificio Barlovento

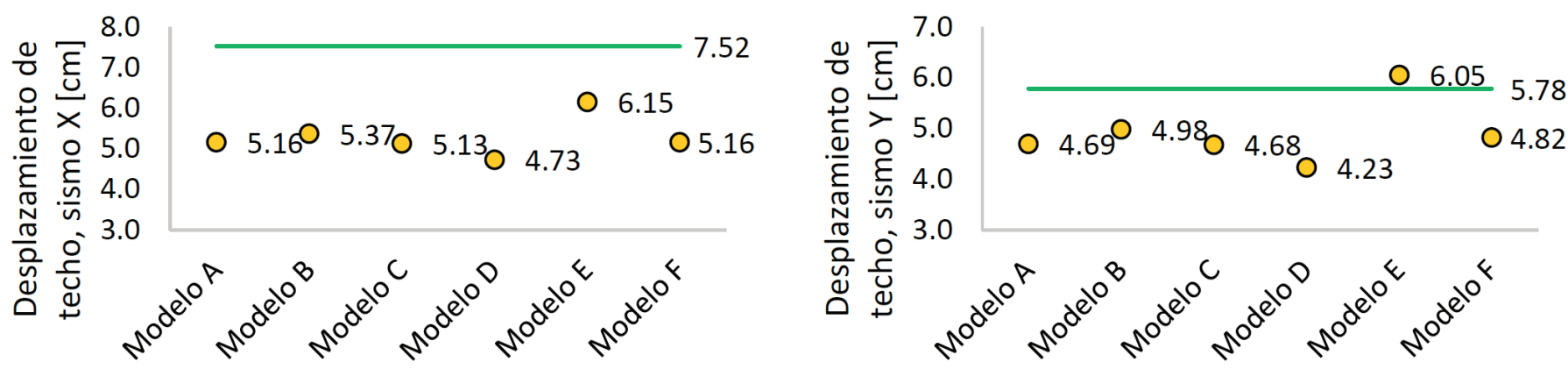

Figura 7: Desplazamiento de techo en el edificio Grecia

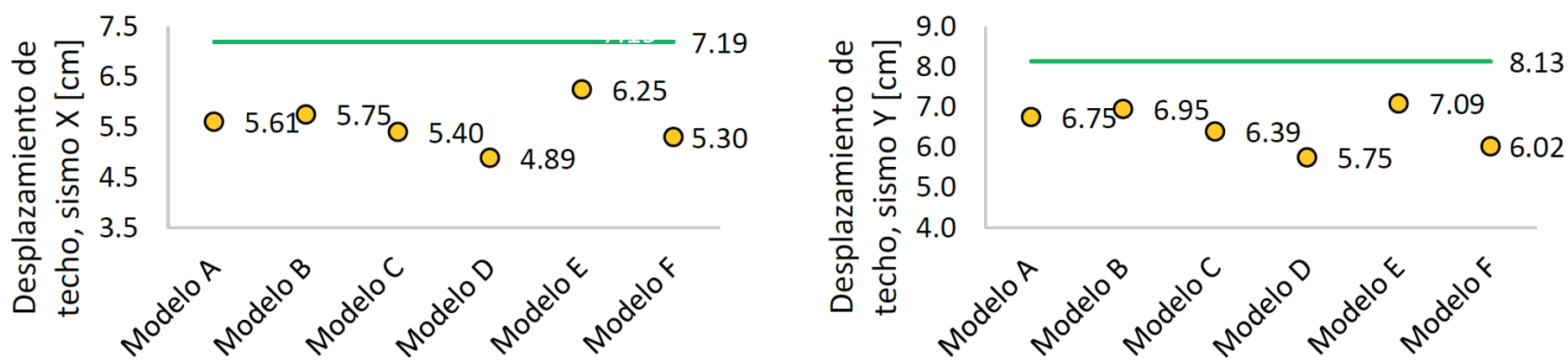

Figura 8: Desplazamiento de techo en el edificio Las Palmas 


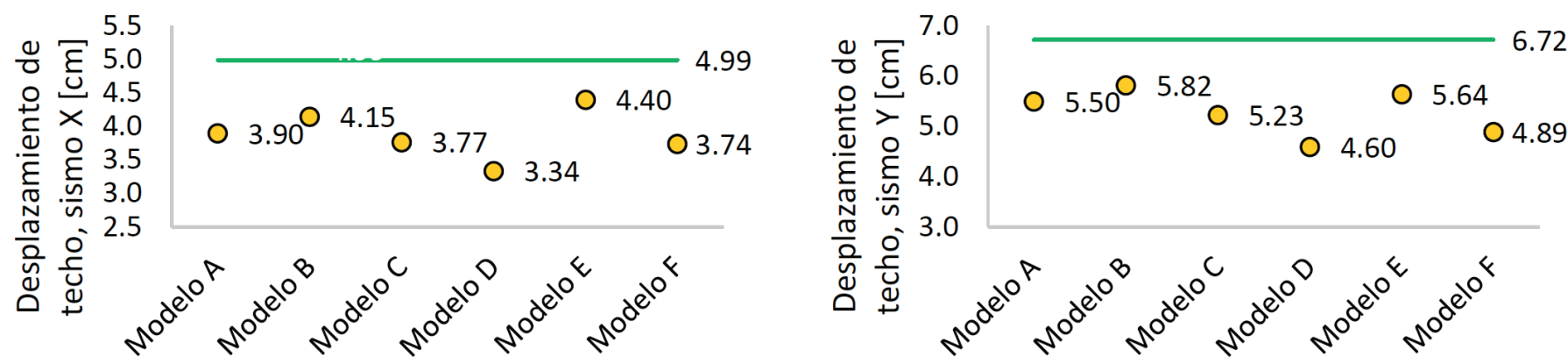

Figura 9: Desplazamiento de techo en el edificio Los Sauces

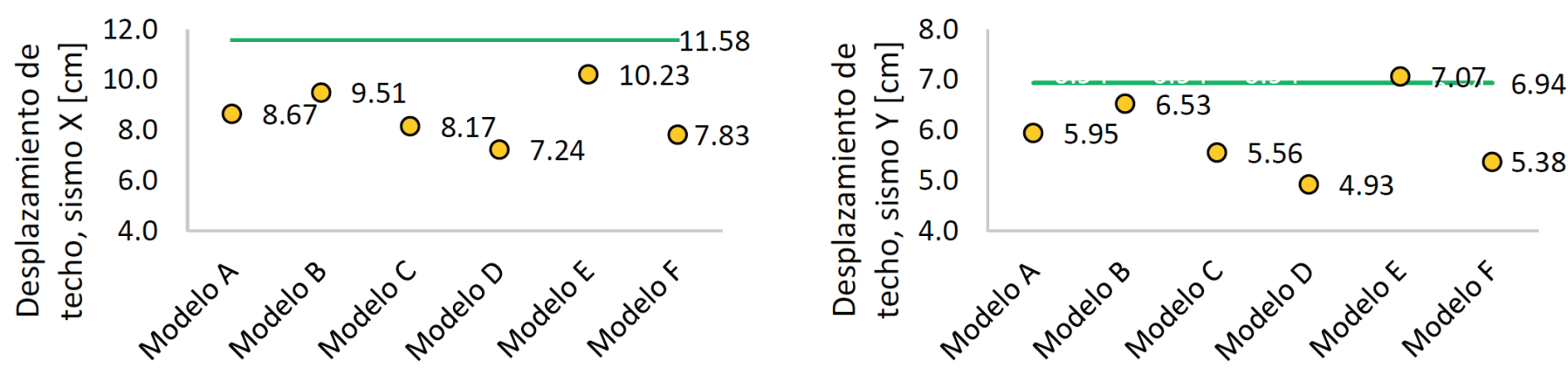

Figura 10: Desplazamiento de techo en el edificio Mar del Sur

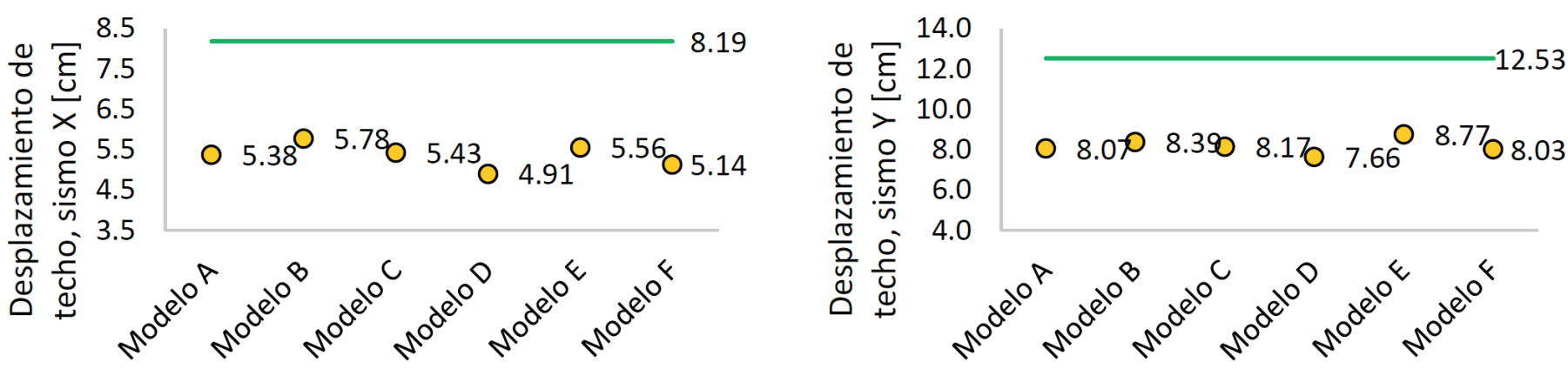

Figura 11: Desplazamiento de techo en el edificio Mirador del Sur

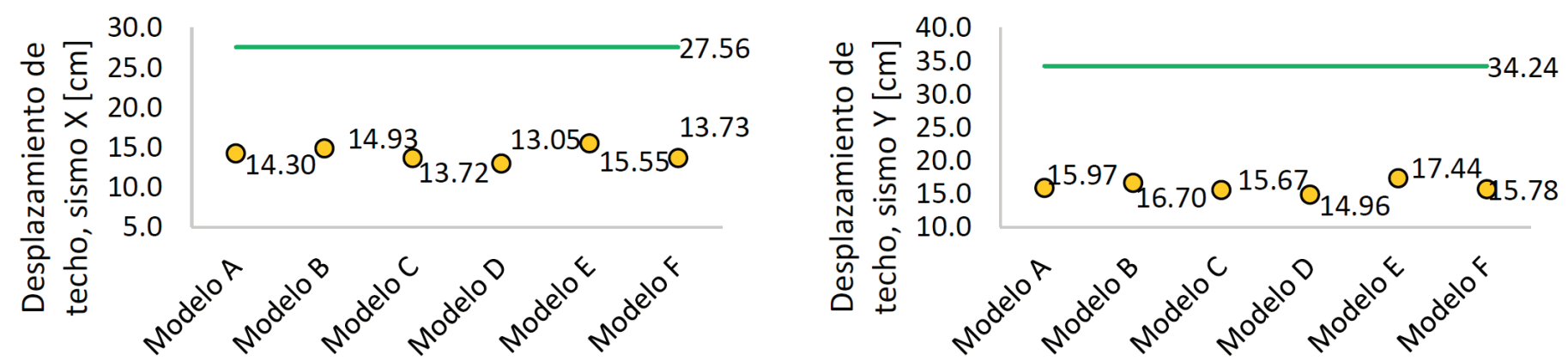

Figura 12: Desplazamiento de techo en el edificio Pérez Zujovic 

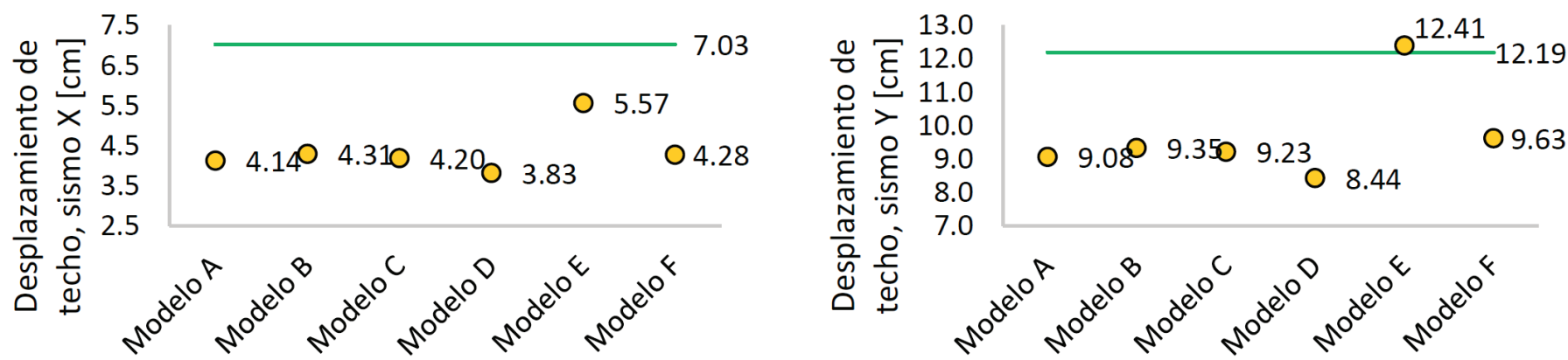

Figura 13: Desplazamiento de techo en el edificio Plaza Foresta

De los resultados (que se resumen en la Tabla 8) se tiene que en general los desplazamientos de techo determinados con los modelos de agrietamiento no sobrepasan al desplazamiento dado por el D.S. 61, excepto para el modelo $\mathrm{E}$ en algunos edificios.

Tabla 8: Desplazamiento de techo caso agrietado/desplazamiento según D.S61 (en \%) para los distintos modelos

\begin{tabular}{|c|c|c|c|c|c|c|c|}
\hline \multirow{2}{*}{ Edificio } & Dirección & $\mathrm{A}$ & $\mathrm{B}$ & $\mathrm{C}$ & $\mathrm{D}$ & $\mathrm{E}$ & $\mathrm{F}$ \\
\hline \multirow{2}{*}{ Barlovento } & $X$ & 62 & 66 & 63 & 58 & 88 & 66 \\
\cline { 2 - 8 } & $Y$ & 78 & 82 & 78 & 70 & 109 & 76 \\
\hline \multirow{3}{*}{ Grecia } & $X$ & 69 & 71 & 68 & 63 & 82 & 69 \\
\cline { 2 - 8 } & $Y$ & 81 & 86 & 81 & 73 & 105 & 83 \\
\hline \multirow{3}{*}{ Las Palmas } & $X$ & 78 & 80 & 75 & 68 & 87 & 74 \\
\cline { 2 - 8 } & $Y$ & 83 & 85 & 79 & 71 & 87 & 74 \\
\hline \multirow{2}{*}{ Mar del Sur } & $X$ & 78 & 83 & 76 & 67 & 88 & 75 \\
\cline { 2 - 8 } & $Y$ & 82 & 87 & 78 & 68 & 84 & 73 \\
\hline \multirow{2}{*}{ Mirador del Sur } & $X$ & 75 & 72 & 71 & 63 & 88 & 68 \\
\cline { 2 - 8 } & $Y$ & 66 & 81 & 80 & 71 & 102 & 78 \\
\hline \multirow{2}{*}{ Perez Zujovic } & $X$ & 52 & 54 & 50 & 47 & 56 & 50 \\
\cline { 2 - 8 } & $Y$ & 47 & 49 & 46 & 44 & 51 & 46 \\
\hline \multirow{2}{*}{ Plaza Foresta } & $X$ & 59 & 61 & 60 & 54 & 79 & 61 \\
\cline { 2 - 8 } & $Y$ & 74 & 77 & 76 & 69 & 102 & 79 \\
\hline
\end{tabular}

\section{Período y desplazamiento de techo según niveles de piso con muros agrietados}

Con el objetivo de estudiar la variación del período agrietado y del desplazamiento de techo al considerar distintos niveles de piso con muros agrietados, a continuación se muestran los resultados obtenidos para dos, tres y cuatro niveles de pisos agrietado para todos los edificios, con excepción del edificio Pérez Zujovic, al cual se le consideró sus primeros cuatro, cinco y seis niveles con muros agrietados. Lo antes señalado, es aplicado considerando los modelos A, B, C y $\mathrm{D}$ dado que los modelos $\mathrm{E}$ y $\mathrm{F}$ modifican la rigidez por un único factor a lo largo de todo el muro. Las Figuras 14 a 18 muestran la relación $k$ de período agrietado/período no agrietado en $X$ e $Y$.

Los resultados se resumen en las Tablas 9 a 13, en las cuales se presenta en la primera columna el valor de $k$ para tres niveles de agrietamiento en todos los edificios y cinco para el edificio Pérez Zujovic y en las columnas posteriores los resultados de $k$ para los dos casos más analizados, con su respectivo porcentaje de variación en relación al de tres o cinco niveles, según corresponda.

Tabla 9: Valores de $k$ en edificios en que se consideró 2, 3 y 4 niveles de pisos agrietados, según modelo A

\begin{tabular}{|l|c|c|c|c|c|c|c|c|c|c|}
\hline \multirow{2}{*}{ Edificios } & \multicolumn{3}{|c|}{ Sismo dirección $X$} & \multicolumn{5}{c|}{ Sismo dirección $Y$} \\
\cline { 2 - 12 } & 3 & \multicolumn{2}{|c|}{$\begin{array}{c}2 \\
k \mathrm{y} \%\end{array}$} & $\begin{array}{c}4 \\
k \mathrm{y} \%\end{array}$ & 3 & \multicolumn{2}{|c|}{$\begin{array}{c}2 \\
k \mathrm{y} \%\end{array}$} & \multicolumn{2}{|c|}{$\begin{array}{c}4 \\
k \mathrm{y} \%\end{array}$} \\
\hline Barlovento & 1.19 & 1.17 & 98 & 1.24 & 104 & 1.27 & 1.22 & 96 & 1.33 & 105 \\
\hline Grecia & 1.26 & 1.16 & 92 & 1.29 & 103 & 1.40 & 1.30 & 93 & 1.44 & 103 \\
\hline Las Palmas & 1.39 & 1.29 & 93 & 1.40 & 101 & 1.41 & 1.34 & 95 & 1.45 & 103 \\
\hline Los Sauces & 1.41 & 1.35 & 96 & 1.49 & 106 & 1.45 & 1.41 & 97 & 1.53 & 106 \\
\hline $\begin{array}{l}\text { Mar del } \\
\text { Sur }\end{array}$ & 1.29 & 1.26 & 98 & 1.35 & 105 & 1.42 & 1.35 & 95 & 1.48 & 104 \\
\hline $\begin{array}{l}\text { Mirador del } \\
\text { Sur }\end{array}$ & 1.68 & 1.64 & 98 & 1.75 & 104 & 1.21 & 1.19 & 99 & 1.28 & 106 \\
\hline $\begin{array}{l}\text { Plaza } \\
\text { Foresta }\end{array}$ & 1.18 & 1.14 & 97 & 1.23 & 104 & 1.32 & 1.25 & 95 & 1.39 & 105 \\
\hline
\end{tabular}



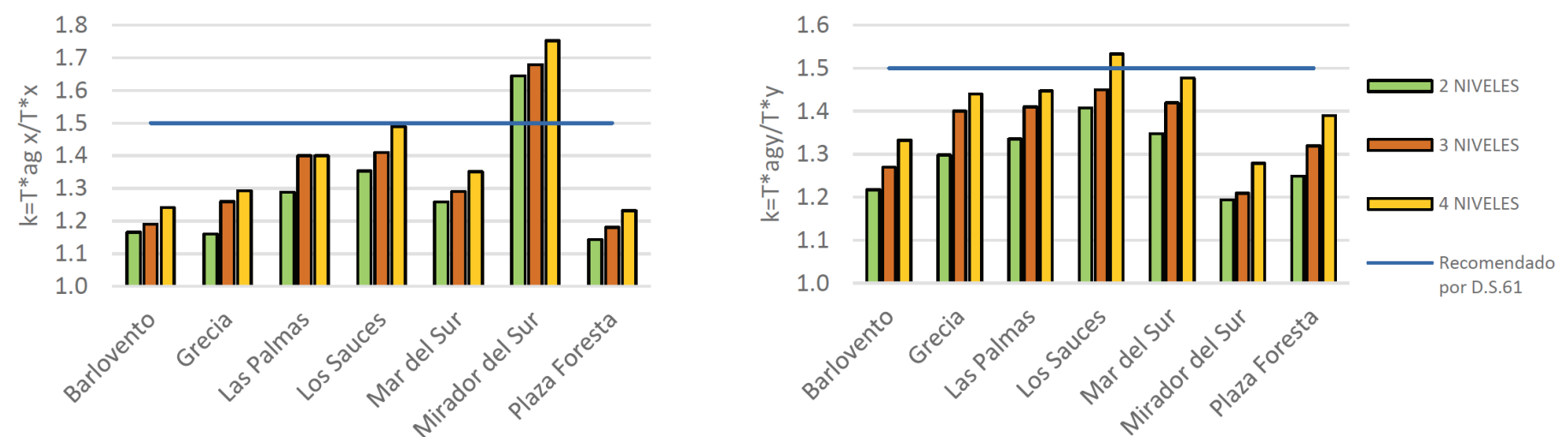

Figura 14: Valores de $k$ según modelo A para edificios en que se consideraron 2, 3 y 4 niveles de muros agrietados
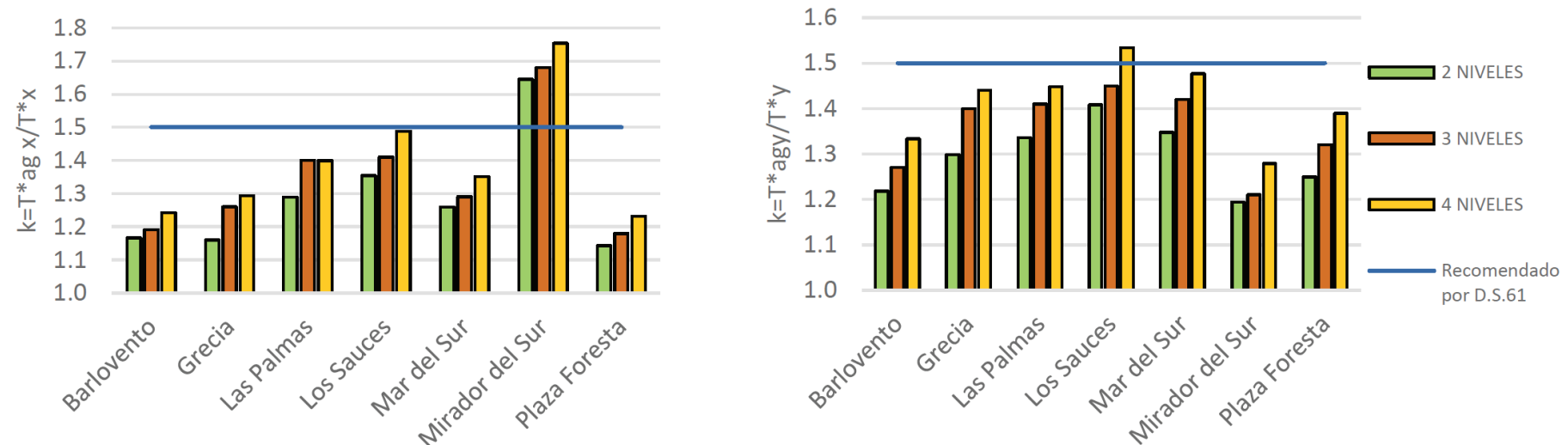

Figura 15: Valores de $k$ según modelo B para edificios en que se consideraron 2, 3 y 4 niveles de muros agrietados
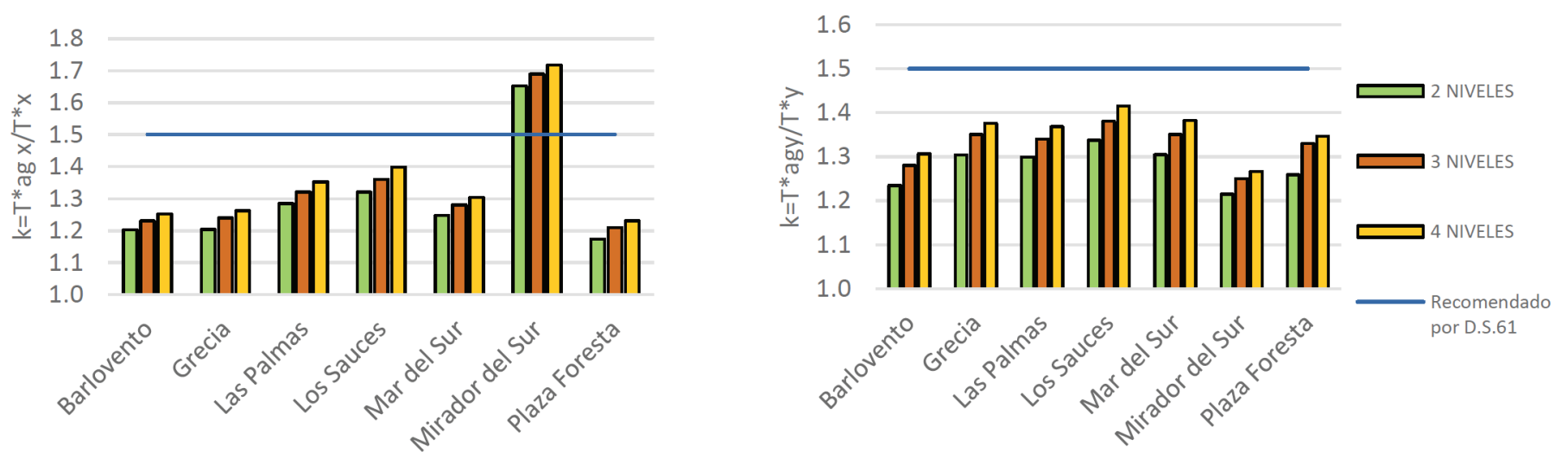

Figura 16: Valores de $k$ según modelo $C$ para edificios en que se consideraron 2, 3 y 4 niveles de muros agrietados
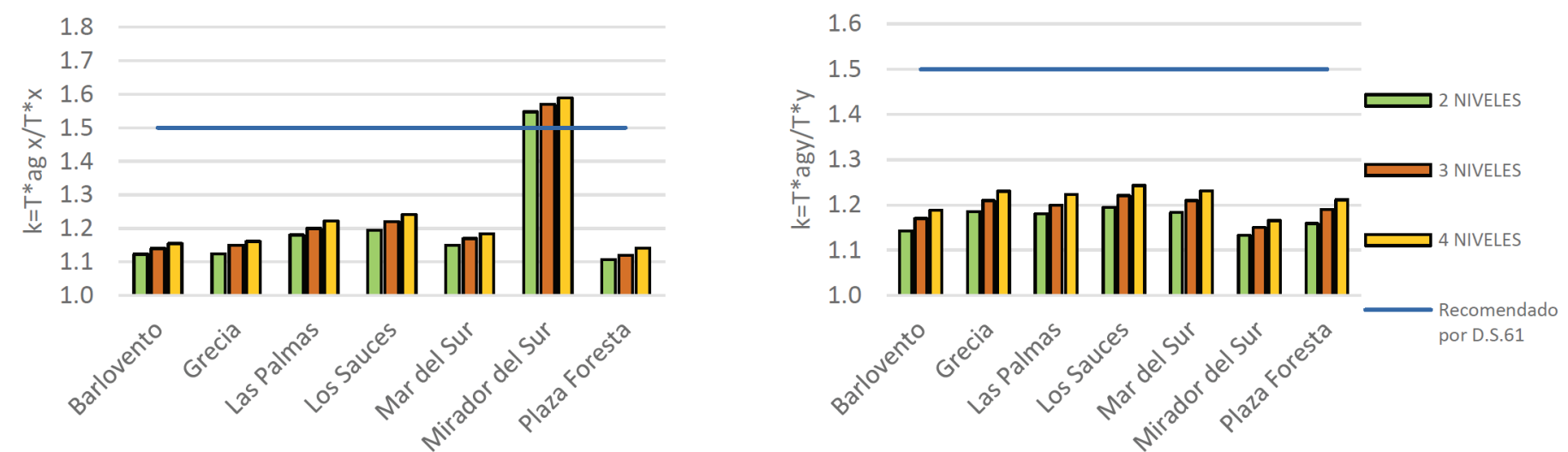

Figura 17: Valores de $k$ según modelo D para edificios en que se consideraron 2, 3 y 4 niveles de muros agrietados 

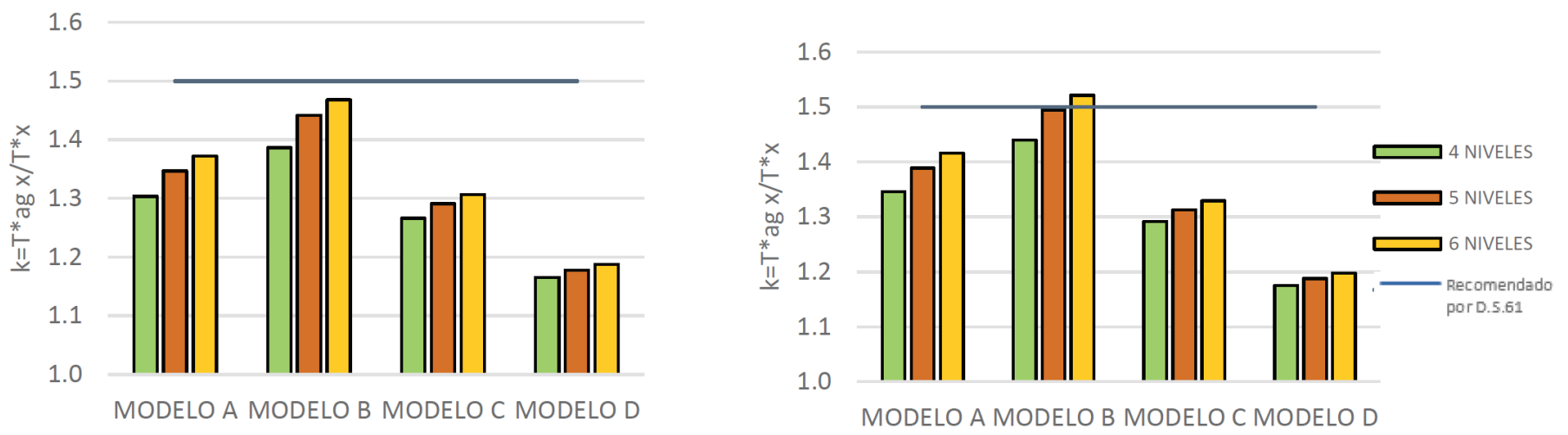

Figura 18: Valores de $k$ para edificio Pérez Zujovic considerando 4, 5 y 6 niveles de muros agrietados

Tabla 10: Valores de $k$ en edificios en que se consideró 2, 3 y 4 niveles de pisos agrietados, según modelo B

\begin{tabular}{|l|c|c|c|c|c|c|c|c|c|c|}
\hline \multirow{2}{*}{ Edificios } & \multicolumn{4}{|c|}{ Sismo dirección $X$} & \multicolumn{4}{c|}{ Sismo dirección $Y$} \\
\cline { 2 - 11 } & 3 & \multicolumn{2}{|c|}{$\begin{array}{c}2 \\
k \mathrm{y} \%\end{array}$} & \multicolumn{2}{c|}{$\begin{array}{c}4 \\
k \mathrm{y} \%\end{array}$} & 3 & $\begin{array}{c}2 \\
k \mathrm{y} \%\end{array}$ & \multicolumn{2}{|c|}{$\begin{array}{c}4 \\
k \mathrm{y} \%\end{array}$} \\
\hline Barlovento & 1.25 & 1.23 & 99 & 1.32 & 105 & 1.33 & 1.29 & 97 & 1.42 & 106 \\
\hline Grecia & 1.29 & 1.25 & 97 & 1.35 & 105 & 1,47 & 1.40 & 95 & 1.53 & 104 \\
\hline Las Palmas & 1.41 & 1.33 & 94 & 1.47 & 104 & 1.44 & 1.31 & 91 & 1.50 & 104 \\
\hline Los Sauces & 1.50 & 1.45 & 97 & 1.60 & 106 & 1.53 & 1.51 & 99 & 1.64 & 107 \\
\hline $\begin{array}{l}\text { Mar del } \\
\text { Sur }\end{array}$ & 1.37 & 1.35 & 98 & 1.46 & 106 & 1.53 & 1.46 & 95 & 1.61 & 105 \\
\hline $\begin{array}{l}\text { Mirador } \\
\text { del Sur }\end{array}$ & 1.78 & 1.73 & 97 & 1.85 & 104 & 1.30 & 1.26 & 97 & 1.35 & 104 \\
\hline $\begin{array}{l}\text { Plaza } \\
\text { Foresta }\end{array}$ & 1.21 & 1.19 & 98 & 1.27 & 105 & 1.36 & 1.30 & 96 & 1.42 & 105 \\
\hline
\end{tabular}

Tabla 11: Valores de $k$ en edificios en que se consideró 2, 3 y 4 niveles de pisos agrietados, según modelo $\mathrm{C}$

\begin{tabular}{|l|c|c|c|c|c|c|c|c|c|c|}
\hline \multirow{2}{*}{ Edificios } & \multicolumn{4}{|c|}{ Sismo dirección $X$} & \multicolumn{4}{c|}{ Sismo dirección $Y$} \\
\cline { 2 - 12 } & 3 & \multicolumn{2}{|c|}{$\begin{array}{c}2 \\
k \mathrm{y} \%\end{array}$} & \multicolumn{2}{c|}{$\begin{array}{c}4 \\
k \mathrm{y}\end{array}$} & 3 & \multicolumn{2}{|c|}{$\begin{array}{c}2 \\
k \mathrm{y} \%\end{array}$} & \multicolumn{2}{c|}{$\begin{array}{c}4 \\
k \mathrm{y} \%\end{array}$} \\
\hline Barlovento & 1.23 & 1.20 & 98 & 1.25 & 102 & 1.28 & 1.23 & 96 & 1.31 & 102 \\
\hline Grecia & 1.24 & 1.20 & 97 & 1.26 & 102 & 1.35 & 1.30 & 97 & 1.38 & 102 \\
\hline $\begin{array}{l}\text { Las } \\
\text { Palmas }\end{array}$ & 1.32 & 1.28 & 97 & 1.35 & 102 & 1.34 & 1.30 & 97 & 1.37 & 102 \\
\hline Los Sauces & 1.36 & 1.32 & 97 & 1.40 & 103 & 1.38 & 1.34 & 97 & 1.41 & 103 \\
\hline $\begin{array}{l}\text { Mar del } \\
\text { Sur }\end{array}$ & 1.28 & 1.25 & 97 & 1.30 & 102 & 1.35 & 1.30 & 97 & 1.38 & 102 \\
\hline $\begin{array}{l}\text { Mirador } \\
\text { del Sur }\end{array}$ & 1.69 & 1.65 & 98 & 1.72 & 102 & 1.25 & 1.21 & 97 & 1.27 & 101 \\
\hline $\begin{array}{l}\text { Plaza } \\
\text { Foresta }\end{array}$ & 1.20 & 1.17 & 98 & 1.23 & 103 & 1.31 & 1.26 & 96 & 1.35 & 103 \\
\hline
\end{tabular}

Tabla 12: Valores de $k$ en edificios en que se consideró 2, 3 y 4 niveles de pisos agrietados, según modelo D

\begin{tabular}{|l|c|c|c|c|c|c|c|c|c|c|}
\hline \multirow{2}{*}{ Edificios } & \multicolumn{4}{|c|}{ Sismo dirección $X$} & \multicolumn{4}{c|}{ Sismo dirección $Y$} \\
\cline { 2 - 12 } & 3 & $\begin{array}{c}2 \\
k \mathrm{y} \%\end{array}$ & $\begin{array}{c}4 \\
k \mathrm{y} \%\end{array}$ & 3 & \multicolumn{2}{c|}{$\begin{array}{c}2 \\
k \mathrm{y} \%\end{array}$} & \multicolumn{2}{c|}{$\begin{array}{c}4 \\
k \mathrm{y} \%\end{array}$} \\
\hline Barlovento & 1.14 & 1.12 & 99 & 1.16 & 101 & 1.17 & 1.14 & 98 & 1.19 & 102 \\
\hline Grecia & 1.15 & 1.12 & 98 & 1.16 & 101 & 1.21 & 1.19 & 98 & 1.23 & 102 \\
\hline Las Palmas & 1.20 & 1.18 & 98 & 1.22 & 102 & 1.20 & 1.18 & 98 & 1.22 & 102 \\
\hline Los Sauces & 1.22 & 1.19 & 98 & 1.24 & 102 & 1.22 & 1.19 & 98 & 1.24 & 102 \\
\hline Mar del Sur & 1.17 & 1.15 & 98 & 1.18 & 101 & 1.21 & 1.18 & 98 & 1.23 & 102 \\
\hline $\begin{array}{l}\text { Mirador del } \\
\text { Sur }\end{array}$ & 1.57 & 1.55 & 99 & 1.59 & 101 & 1.15 & 1.13 & 98 & 1.17 & 101 \\
\hline $\begin{array}{l}\text { Plaza } \\
\text { Foresta }\end{array}$ & 1.12 & 1.11 & 99 & 1.14 & 102 & 1.19 & 1.16 & 97 & 1.21 & 102 \\
\hline
\end{tabular}

Tabla 13: Valores de $k$ en edificio Pérez Zujovic en que se consideró 4, 5 y 6 niveles de pisos agrietados, según modelos A, B, C y D

\begin{tabular}{|c|c|c|c|c|c|c|c|c|c|c|}
\hline \multirow{2}{*}{ Modelos } & \multicolumn{4}{|c|}{ Dirección $X$} & \multicolumn{5}{c|}{ Dirección $Y$} \\
\cline { 2 - 11 } & 5 & $\begin{array}{c}4 \\
k \mathrm{y} \%\end{array}$ & \multicolumn{2}{|c|}{$\begin{array}{c}6 \\
k \mathrm{y} \%\end{array}$} & 5 & \multicolumn{2}{|c|}{$\begin{array}{c}4 \\
k \mathrm{y} \%\end{array}$} & \multicolumn{2}{|c|}{$\begin{array}{c}6 \\
k \mathrm{y} \%\end{array}$} \\
\hline $\mathrm{A}$ & 1.39 & 1.35 & 97 & 1.42 & 102 & 1.35 & 1.30 & 96 & 1.37 & 101 \\
\hline $\mathrm{B}$ & 1.49 & 1.44 & 97 & 1.52 & 102 & 1.44 & 1.39 & 97 & 1.47 & 102 \\
\hline $\mathrm{C}$ & 1.31 & 1.29 & 98 & 1.33 & 102 & 1.29 & 1.27 & 98 & 1.31 & 102 \\
\hline $\mathrm{D}$ & 1.19 & 1.17 & 98 & 1.20 & 101 & 1.18 & 1.17 & 99 & 1.19 & 101 \\
\hline
\end{tabular}

A continuación se resume la variación obtenida de la relación $k$ (en \%) respecto al caso de considerar los tres primeros niveles de pisos agrietados para todos los edificios y los cinco primeros niveles para el edificio Perez Zujovic. 
- Modelo A: para todos los edificios, excepto el Pérez Zujovic, el valor de $k$ considerando dos niveles de pisos agrietados varía hasta $-8 \%$ y para cuatro niveles de pisos agrietados hasta 6\%. En el edificio Pérez Zujovic las variaciones de $k$ son de $-4 \%$ al considerar cuatro niveles y $2 \%$ al considerar seis niveles de pisos agrietados.

- Modelo B: para todos los edificios, excepto el Pérez Zujovic, el valor de $k$ considerando dos niveles de pisos agrietados varía hasta $-9 \%$ y para cuatro niveles de pisos agrietados hasta 7\%. En el edificio Pérez Zujovic las variaciones de $k$ son de $-3 \%$ al considerar cuatro niveles y $2 \%$ al considerar seis niveles de pisos agrietados.

- Modelo C: para todos los edificios, excepto el Pérez Zujovic, el valor de $k$ considerando dos niveles de pisos agrietados varía hasta $-4 \%$ y para cuatro niveles de pisos agrietados hasta $3 \%$. En el edificio Pérez Zujovic las variaciones de $k$ son de $-2 \%$ al considerar cuatro niveles y $2 \%$ al considerar seis niveles de pisos agrietados.

- Modelo D: para todos los edificios, excepto el Pérez Zujovic, el valor de $k$ considerando dos niveles de pisos agrietados varía hasta $-3 \%$ y para cuatro niveles de pisos agrietados hasta $2 \%$. En el edificio Pérez Zujovic las variaciones de $k$ son de $-2 \%$ al considerar cuatro niveles y $1 \%$ al considerar seis niveles de pisos agrietados.

De lo anterior se puede concluir que en los edificios (excepto el edificio Pérez Zujovic) y casos analizados, el considerar dos o cuatro niveles de piso con muros agrietados, implica una variación del factor $k$ inferior a un $10 \%$ respecto a considerar tres niveles. Para el edificio Pérez Zujovic se tiene que al considerar cuatro y seis niveles de piso agrietado provoca una variación del factor $k$ inferior a un 4\%, con respecto a considerar cinco niveles.

\section{Desplazamientos de techo}

En las Tablas 14 a 18 se muestran, para todos los edificios analizados y los modelos A, B, C y D, los valores obtenidos del desplazamiento de techo producto del análisis del caso agrietado considerando distintos niveles de pisos con muros agrietados y el porcentaje de variación de éste en relación al caso referencial (igual a lo descrito en la sección anterior). Además, se muestra el desplazamiento último de techo determinado según el Decreto 61.

Tabla 14: Desplazamiento de techo en edificios en que se consideró 2, 3 y 4 niveles de pisos agrietados, según modelo A

\begin{tabular}{|c|c|c|c|c|c|c|c|c|c|c|c|c|}
\hline \multirow{3}{*}{$\begin{array}{l}\text { Edificios } \\
\text { Barlo- } \\
\text { vento }\end{array}$} & \multicolumn{6}{|c|}{$\begin{array}{c}\text { Desplazamiento para sismo } \\
\qquad X, \mathrm{~cm}\end{array}$} & \multicolumn{6}{|c|}{$\begin{array}{l}\text { Desplazamiento para sismo } \\
\qquad Y, \mathrm{~cm}\end{array}$} \\
\hline & \multirow{2}{*}{\begin{tabular}{|c|}
$\delta_{\mathrm{u}}$ \\
5.3
\end{tabular}} & \multirow{2}{*}{$\begin{array}{c}3 \\
3.3\end{array}$} & \multicolumn{2}{|c|}{$\begin{array}{c}2 \\
k \mathrm{y} \% \\
\end{array}$} & \multicolumn{2}{|c|}{$\begin{array}{c}4 \\
k \mathrm{y} \% \\
\end{array}$} & \multirow{2}{*}{\begin{tabular}{|c|}
$\delta_{\mathrm{u}}$ \\
12.1
\end{tabular}} & \multirow{2}{*}{$\begin{array}{c}3 \\
9.5\end{array}$} & \multicolumn{2}{|c|}{$\begin{array}{c}2 \\
k \mathrm{y} \% \\
\end{array}$} & \multicolumn{2}{|c|}{$\begin{array}{c}4 \\
k \mathrm{y} \% \\
\end{array}$} \\
\hline & & & 3.2 & 97 & 4.0 & 122 & & & 90 & 95 & 11.9 & 126 \\
\hline Grecia & 7.5 & & & & & 102 & 5.8 & 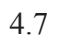 & & 97 & 8 & 103 \\
\hline $\mathrm{L}$ & 7.2 & 5.6 & 5.2 & 92 & 5.7 & 101 & 8.1 & 6.8 & 6.4 & 95 & 7.0 & 103 \\
\hline & 5.0 & 3.9 & 3.8 & 96 & 4.1 & 105 & 6.7 & 5.5 & 5.3 & 97 & 5.8 & 106 \\
\hline 1 & 11.6 & 8.7 & 8.2 & 95 & 9.1 & 105 & 6.9 & 6.0 & 5.5 & 93 & 6.3 & 105 \\
\hline $\mathrm{r}$ & 8.2 & 5.4 & 5.3 & 90 & 6.4 & 118 & 12.5 & 8.1 & 7.8 & 97 & 8.4 & 104 \\
\hline 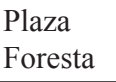 & 7.0 & 4.1 & 4.0 & 97 & 4.4 & 105 & 12.2 & 9.1 & 8.6 & 95 & 10.7 & 118 \\
\hline
\end{tabular}

Tabla 15: Desplazamiento de techo en edificios en que se consideró 2, 3 y 4 niveles de pisos agrietados, según modelo B

\begin{tabular}{|c|c|c|c|c|c|c|c|c|c|c|c|c|}
\hline \multirow{3}{*}{$\begin{array}{l}\text { Edificios } \\
\text { Barlo- } \\
\text { vento }\end{array}$} & \multicolumn{6}{|c|}{$\begin{array}{c}\text { Desplazamiento para nismo } \\
X, \mathrm{~cm}\end{array}$} & \multicolumn{6}{|c|}{$\begin{array}{c}\text { Desplazamiento para sismo } \\
Y, \mathrm{~cm}\end{array}$} \\
\hline & \multirow{2}{*}{$\begin{array}{c}\delta_{\mathrm{u}} \\
5.3\end{array}$} & \multirow{2}{*}{$\begin{array}{c}3 \\
3.5\end{array}$} & \multicolumn{2}{|c|}{$\begin{array}{c}2 \\
k \mathrm{y} \% \\
\end{array}$} & \multicolumn{2}{|c|}{$\begin{array}{c}4 \\
k \mathrm{y} \% \\
\end{array}$} & \multirow{2}{*}{\begin{tabular}{|l|l} 
& $\delta_{\mathrm{u}}$ \\
12.1
\end{tabular}} & \multirow{2}{*}{$\begin{array}{c}3 \\
9.9\end{array}$} & \multicolumn{2}{|c|}{$\begin{array}{c}2 \\
k \mathrm{y} \% \\
\end{array}$} & \multicolumn{2}{|c|}{$\begin{array}{c}4 \\
k \mathrm{y} \% \\
\end{array}$} \\
\hline & & & 3.2 & 91 & 4.3 & 123 & & & 9.6 & 96 & 12.7 & 128 \\
\hline Grecia & & & & & & 3 & 8 & 5.0 & 1.7 & 95 & 1 & 10 \\
\hline & 7.2 & 5.8 & 5.4 & 95 & 6.0 & 104 & 8.1 & 7.0 & 6.3 & 90 & 7.2 & 103 \\
\hline & 5.0 & 4.2 & 4.0 & 97 & 4.4 & 106 & 6.7 & 5.8 & 5.7 & 98 & 6.2 & 107 \\
\hline ur & 11.6 & 9.5 & 9.0 & 95 & 10.1 & 106 & 6.9 & 6.5 & 6.0 & 92 & 6.9 & 106 \\
\hline & 8.2 & 5.8 & 5.7 & 98 & 6.9 & 119 & 12.5 & 8.4 & 8.2 & 98 & 9.0 & 107 \\
\hline 1016 & 7.0 & 4.3 & 4.2 & 98 & 4.5 & 105 & 12.2 & 9.4 & 9.0 & 97 & 11.0 & 118 \\
\hline
\end{tabular}


Tabla 16: Desplazamiento de techo en edificios en que se consideró 2, 3 y 4 niveles de pisos agrietados, según modelo $\mathrm{C}$.

\begin{tabular}{|c|c|c|c|c|c|c|c|c|c|c|c|c|}
\hline \multirow{3}{*}{$\begin{array}{c}\text { Edificios } \\
\text { Barlovento }\end{array}$} & \multicolumn{6}{|c|}{$\begin{array}{c}\text { Desplazamiento para sismo } \\
\qquad X, \mathrm{~cm}\end{array}$} & \multicolumn{6}{|c|}{$\begin{array}{c}\text { Desplazamiento para sismo } \\
\qquad Y, \mathrm{~cm}\end{array}$} \\
\hline & \multirow{2}{*}{\begin{tabular}{|c|}
$\delta_{\mathrm{u}}$ \\
5.3 \\
\end{tabular}} & \multirow{2}{*}{$\begin{array}{c}3 \\
3.4 \\
\end{array}$} & \multicolumn{2}{|c|}{$\begin{array}{c}2 \\
k \mathrm{y} \%\end{array}$} & \multicolumn{2}{|c|}{$\begin{array}{c}4 \\
k \mathrm{y} \% \\
\end{array}$} & \multirow{2}{*}{\begin{tabular}{|c|}
$\delta_{\mathrm{u}}$ \\
12.1 \\
\end{tabular}} & \multirow{2}{*}{$\begin{array}{c}3 \\
9.5 \\
\end{array}$} & \multicolumn{2}{|c|}{$\begin{array}{c}2 \\
k \mathrm{y} \%\end{array}$} & \multicolumn{2}{|c|}{$\begin{array}{c}4 \\
k \mathrm{y} \%\end{array}$} \\
\hline & & & 3.3 & 97 & 4.0 & 119 & & & 9.0 & 96 & 11.5 & 122 \\
\hline Grecia & 7.5 & 5.1 & 5.0 & 97 & 5.2 & 102 & 5.8 & 4.7 & 4.5 & 97 & 4.8 & 102 \\
\hline $\begin{array}{l}\text { Las Pal- } \\
\text { mas }\end{array}$ & 7.2 & 5.4 & 5.2 & 97 & 5.5 & 102 & 8.1 & 6.4 & 6.2 & 97 & 6.6 & 103 \\
\hline Los Sauces & 5.0 & & 3.7 & 7 & 3.9 & 102 & 6.7 & 5.2 & 5.1 & 97 & 5.4 & 103 \\
\hline $\begin{array}{l}\text { Mar } \\
\text { Sur }\end{array}$ & 11.6 & 8.2 & 7.9 & 97 & 8.9 & 109 & 6.9 & 5.6 & 5.3 & 96 & 6.8 & 122 \\
\hline $\begin{array}{l}\text { Mirador } \\
\text { del Sur }\end{array}$ & 8.2 & 5.4 & 5.3 & 97 & 5.5 & 102 & 12.5 & 8.2 & 8.0 & 98 & 8.3 & 101 \\
\hline $\begin{array}{l}\text { Plaza } \\
\text { Foresta }\end{array}$ & 7.0 & 4.2 & 4.1 & 98 & 4.4 & 104 & 12.2 & 9.2 & 8.9 & 96 & 10.7 & 115 \\
\hline
\end{tabular}

Tabla 17: Desplazamiento de techo en edificios en que se consideró 2, 3 y 4 niveles de pisos agrietados, según modelo D.

\begin{tabular}{|c|c|c|c|c|c|c|c|c|c|c|c|c|}
\hline \multirow{3}{*}{$\begin{array}{l}\text { Edificios } \\
\text { Barlovento }\end{array}$} & \multicolumn{6}{|c|}{$\begin{array}{c}\text { Desplazamiento para sismo } \\
X, \mathrm{~cm}\end{array}$} & \multicolumn{6}{|c|}{$\begin{array}{c}\text { Desplazamiento para sismo } \\
Y, \mathrm{~cm}\end{array}$} \\
\hline & \multirow{2}{*}{\begin{tabular}{|c|}
$\delta_{u}$ \\
5.3 \\
\end{tabular}} & \multirow{2}{*}{$\begin{array}{c}3 \\
3.1 \\
\end{array}$} & \multicolumn{2}{|c|}{$\begin{array}{c}2 \\
k \mathrm{y} \% \\
\end{array}$} & \multicolumn{2}{|c|}{$\begin{array}{c}4 \\
k \mathrm{y} \% \\
\end{array}$} & \multirow{2}{*}{\begin{tabular}{|c|}
$\delta_{u}$ \\
12.1 \\
\end{tabular}} & \multirow{2}{*}{$\begin{array}{c}3 \\
8.5 \\
\end{array}$} & \multicolumn{2}{|c|}{\begin{tabular}{|c|}
2 \\
$k \mathrm{y} \%$
\end{tabular}} & \multicolumn{2}{|c|}{$\begin{array}{c}4 \\
k \mathrm{y} \%\end{array}$} \\
\hline & & & 3.0 & 98 & 3.6 & 118 & & & 8.2 & 97 & 10.2 & 121 \\
\hline ecia & 75 & 4 & 4.6 & 8 & 8 & 101 & 5.8 & 4.2 & 4.1 & 98 & 4.3 & 102 \\
\hline Las Palmas & 7.2 & 4.9 & 4.8 & 98 & 5.0 & 102 & 8.1 & 5.8 & 5.6 & 98 & 5.8 & 102 \\
\hline Los & 5 & 3.3 & 3.3 & 8 & 4 & 102 & 6.7 & 6 & 4.5 & \begin{tabular}{|l}
98 \\
\end{tabular} & .7 & 102 \\
\hline $\begin{array}{l}\text { Mar } \\
\text { Sur }\end{array}$ & 1.6 & 7.2 & 7.1 & 98 & 6.1 & 84 & 6.9 & 4.9 & 4.8 & 97 & 5.0 & 102 \\
\hline $\begin{array}{l}\text { Mirador } \\
\text { del Sur }\end{array}$ & 8.2 & 4.9 & 4.8 & 98 & 5.5 & 112 & 12.5 & 7.7 & 7.6 & 99 & 7.9 & 103 \\
\hline oresta & 7.0 & 3.8 & 3.8 & 98 & 4.0 & 103 & 12.2 & 8.4 & 8.2 & 98 & 9.6 & 114 \\
\hline
\end{tabular}

Tabla 18: Desplazamiento de techo en edificio Perez Zujovic considerando 4, 5 y 6 niveles de pisos agrietados, según modelos A, B, C y D.

\begin{tabular}{|c|c|c|c|c|c|c|c|c|c|c|c|}
\hline \multirow{2}{*}{ Modelos } & \multicolumn{3}{|c|}{$\begin{array}{c}\text { Desplazamiento para } \\
\text { sismo } X, \mathrm{~cm}\end{array}$} & $\begin{array}{c}\delta_{\mathrm{u}}= \\
27.6 \\
\mathrm{~cm}\end{array}$ & \multicolumn{3}{|c|}{$\begin{array}{c}\text { Desplazamiento para } \\
\text { sismo } Y, \mathrm{~cm}\end{array}$} & $\begin{array}{c}\delta_{\mathrm{u}}= \\
34.2 \\
\mathrm{~cm}\end{array}$ \\
\cline { 2 - 12 } & $\begin{array}{c}5 \\
\text { niveles }\end{array}$ & $\begin{array}{c}4 \text { niveles } \\
\mathrm{k} \mathrm{y} \%\end{array}$ & $\begin{array}{c}6 \text { niveles } \\
\mathrm{k} \text { y } \%\end{array}$ & $\begin{array}{c}5 \\
\text { niveles }\end{array}$ & $\begin{array}{c}4 \text { niveles } \\
\mathrm{k} \mathrm{y} \%\end{array}$ & $\begin{array}{c}6 \text { niveles } \\
\mathrm{k} \mathrm{y} \%\end{array}$ \\
\hline $\mathrm{A}$ & 14.3 & 14.0 & 98 & 14.5 & 102 & 16.0 & 15.7 & 99 & 16.1 & 101 \\
\hline $\mathrm{B}$ & 14.9 & 14.5 & 97 & 15.1 & 101 & 16.7 & 16.3 & 97 & 16.9 & 101 \\
\hline $\mathrm{C}$ & 13.7 & 13.7 & 100 & 13.9 & 101 & 15.7 & 15.6 & 99 & 15.8 & 101 \\
\hline $\mathrm{D}$ & 13.1 & 13.0 & 100 & 13.1 & 101 & 15.0 & 14.9 & 99 & 15.0 & 101 \\
\hline
\end{tabular}

En resumen se tiene lo siguiente:

- Modelo A: para todos los edificios, excepto el Pérez Zujovic, el desplazamiento considerando dos niveles de pisos agrietados varía hasta $-8 \%$ y para cuatro niveles de pisos agrietados hasta $26 \%$. En el edificio Pérez Zujovic el desplazamiento varía hasta un $-2 \%$ al considerar cuatro niveles y $2 \%$ al considerar seis niveles de pisos agrietados.

- Modelo B: para todos los edificios, excepto el Pérez Zujovic, el desplazamiento considerando dos niveles de pisos agrietados varía hasta $-10 \%$ y para cuatro niveles de pisos agrietados hasta $28 \%$. En el edificio Pérez Zujovic el desplazamiento varía hasta un $-3 \%$ al considerar cuatro niveles y $1 \%$ al considerar seis niveles de pisos agrietados.

- Modelo C: para todos los edificios, excepto el Pérez Zujovic, el desplazamiento considerando dos niveles de pisos agrietados varía hasta $-4 \%$ y para cuatro niveles de pisos agrietados hasta $22 \%$. En el edificio Pérez Zujovic el desplazamiento varía hasta un $-1 \%$ al considerar cuatro niveles y $1 \%$ al considerar seis niveles de pisos agrietados.

- Modelo D: para todos los edificios excepto el Pérez Zujovic, el desplazamiento considerando dos niveles de pisos agrietados varía hasta $-3 \%$ y para cuatro niveles de pisos agrietados hasta $21 \%$. En el edificio Pérez Zujovic el desplazamiento varía hasta un $-1 \%$ al considerar cuatro niveles y $1 \%$ al considerar seis niveles de pisos agrietados.

De los resultados obtenidos, además se puede observar que en ningún caso el desplazamiento de techo determinado según los modelos de agrietamiento considerados supera el calculado por el Decreto 61.

\section{Conclusiones}

De los edificios analizados y modelos de agrietamiento considerados en este estudio se puede concluir que la expresión dada por el Decreto 61 para determinar el período de mayor masa traslacional en condiciones agrietada, a partir del período determinado en condiciones no agrietado da en casi todos los edificios y modelos analizados, con excepción el modelo E de Doepker, una relación entre ellos inferior a 1.5. Esto significa que su uso, en estos edificios, implicaría considerar un desplazamiento lateral de diseño en el techo $\delta_{u}$ mayor y por lo tanto una mayor demanda de curvatura a considerar en el diseño de los muros de hormigón armado, todo lo cual implicaría obtener diseños por el lado de la seguridad.

En relación a los desplazamientos de techo, considerando 
en el análisis todos los modelos y tres niveles de pisos con muros agrietados (cinco en el edificio Pérez Zujovic), se tiene que en todos los edificios analizados, con excepción de los analizados con el modelo E de Doepker, los desplazamientos determinados considerando el edificio agrietado son inferiores al desplazamiento calculado según lo indicado por el Decreto 61. Luego, el uso de dicho decreto produciría diseños de muros más conservadores y por el lado de la seguridad.

En relación a los cinco modelos estudiados, se sugiere por simplicidad de aplicación utilizar el modelo $\mathrm{C}$ y F, ambos del código ACI 318, utilizando un factor reductor de 0.35 para muros agrietados y 0.7 para muros no agrietados, o 0.5 en todos los muros. Los modelos de Paulay y Adebar hacen más compleja la determinación del momento efectivo de inercia, ya que requieren la carga axial del muro. Por otro lado, los modelos de Doepker y el FEMA 356 utilizan factores reductores que podrían sobrestimar o subestimar el período agrietado, respectivamente.

Respecto al análisis con variación de niveles de pisos agrietados (para los modelos A, B, C y D), se tiene que en todos los edificios que fueron considerados con dos o cuatro niveles de pisos con muros agrietados, el factor $k$ varía en menos de un $10 \%$ y el desplazamiento de techo es menor al $28 \%$ respecto a considerar tres niveles. Para el edificio Pérez Zujovic al variar a cuatro o seis niveles de pisos con muros agrietados, se obtiene una variación del factor $k$ inferior a $4 \%$ y para el desplazamiento de techo no mayor a $3 \%$ respecto a considerar cinco niveles. Además, el desplazamiento de techo obtenido no supera el determinado por el Decreto 61 en todos los edificios, excepto el edificio Barlovento para el modelo B y sismo en la dirección $Y$, donde resulta levemente mayor. Es importante señalar que el número de niveles de pisos agrietados a considerar en un edificio depende de la ubicación de la sección crítica de cada muro y del cumplimiento de las disposiciones sobre la extensión en altura de ella que establece el código ACI 318 (2008).

\section{Referencias}

ACI 318 (2008). Building code requirements for structural concrete and commentary. American Concrete Institute, USA

ACI 318 (2014). Building code requirements for structural concrete and commentary. American Concrete Institute, USA
Adebar, P. and Ibrahim, A.M. (2002). Simple nonlinear flexural stiffness model for concrete structural walls. Earthquake Spectra 18(3), 407-426

Alfaro, I. (2013). Estimación del desplazamiento lateral elástico e inelástico de muros esbeltos mediante un modelo de rótula plástica basado en un modelo de fibra. Memoria de título, Universidad de Chile.

Cornejo, F. (2012). Desarrollo de una metodología y herramientas de apoyo para el análisis de edificios en altura, de hormigón armado, utilizando programa ETABS. Memoria de título, Universidad Católica del Norte, Antofagasta.

Doepker, B. (2008). Practical seismic analysis methods for structural wall buildings. Master thesis, University of Washington, Seattle.

FEMA 356 (2000). Prestandard and commentary for the seismic rehabilitation of buildings. Federal Emergency Management Agency, Washington, DC

INN (2010). Diseño Sísmico de Edificios. Norma Chilena Oficial NCh433 Of.1996 Mod. 2009 y Decreto Supremo 61. Instituto Nacional de Normalización, Santiago.

Lagos, R., Kupfer, M., Lindenberg, J., Bonelli, P., Saragoni, R., Guendelman, T., Massone, L., Boroschek, R. and Yañez, F. (2012). Seismic performance of high-rise concrete buildings in Chile. International Journal of High-Rise Buildings 1(3), 181-194

MINVU (2011a). Decreto 117. Diseño sísmico de edificios. Ministerio de Vivienda y Urbanismo, Santiago.

MINVU (2011b). Decreto 118. Requisitos para diseño y cálculo de hormigón armado. Ministerio de Vivienda y Urbanismo, Santiago.

MINVU (2011c). Decreto 60. Diseño y cálculo de hormigón armado. Ministerio de Vivienda y Urbanismo, Santiago.

MINVU (2011d). Decreto 61. Diseño sísmico de edificios. Ministerio de Vivienda y Urbanismo, Santiago.

Paulay, T. and Priestley, M.J.N. (1992). Seismic design of reinforced concrete and masonry concrete buildings. John Wiley and Sons, New York

Ríos, H. (2006). Perfil Bio-Sísmico de edificios representativos de la construcción en altura de la ciudad de Antofagasta. Memoria de título, Universidad Católica del Norte, Antofagasta.

Wallace, J.W., Massone, L.M., Bonelli, P., Dragovich, J., Lagos, R., Lüders, C. and Moehle, J. (2012). Damage and implications for seismic design of RC structural wall buildings. Earthquake Spectra 28(S1), S281-S299 